\title{
Time-Hopping Correlation Property and Its Effects on THSS-UWB System
}

\author{
Zhenyu Zhang1,2, Fanxin Zeng'2, Lijia Ge² and Guixin Xuan² \\ ${ }^{1}$ College of Communication Engineering, Chongqing University \\ ${ }^{2}$ Chongqing Communication Institute
}

China

\section{Introduction}

Ultra wideband (UWB) is a promising technology for short-range wireless communications since it potentially combines the reduced complexity with low power consumption, low probability of detection/intercept (LPD/LPI) and immunity to multipath fading (Scholtz, 1993; Win \& Scholtz, 1998; Win \& Scholtz, 2000). The successful development of UWB technology strongly depends on the advancement of efficient multiple-access techniques. A typical multiple-access format of UWB is time-hopping spread spectrum ultra wideband (THSS-UWB) where data are transmitted by using pulse position modulation (PPM) (Scholtz, 1993) or pulse amplitude modulation (PAM) (Le Martret \& Giannakis, 2000) at a rate of many pulses per data symbol. Both of PPM and PAM require good properties of TH sequences.

In terms of $\mathrm{TH}$ correlation properties, the known constructions of $\mathrm{TH}$ sequences can be mainly classified into two categories, namely aperiodic $\mathrm{TH}$ sequences and periodic $\mathrm{TH}$ sequences. For aperiodic TH sequences, the chaotic pulse-position modulation (CPPM) which was designed for the transmission of binary information (Sushchick et al, 2000) and the pseudo-chaotic time hopping (PCTH) which exploited the symbolic dynamics of a chaotic map at the transmitter (Maggio et al, 1999; Maggio et al, 2001) were proposed, respectively. In addition, an alternative use of pseudo-chaotic dynamics was proposed as an encoder for the binary data stream while data-independent TH sequences were used to guarantee ease of synchronization and decidability (Erseghe \& Bramante, 2002). For periodic TH sequences, the related analyses can be found in the corresponding literatures (Chu \& Colbourn, 2004; Corrada-Bravo, 1999; Erseghe, 2002a; Erseghe, 2002b; Iacobucci \& Di Benedetto, 2001; Iacobucci \& Di Benedetto, 2002; Scholtz et al, 2001). These sequences can be considered as the extensions of existing frequency-hopping (FH) sequences and all of them have good $\mathrm{TH}$ correlation properties. In these $\mathrm{TH}$ sequences, quadratic congruence code (QCC) (Scholtz et al, 2001) is the best one in terms of TH correlation properties since QCC satisfies $S_{\max } \leq 2$ and $C_{\max } \leq 4$, where $S_{\max }$ denotes the maximal $\mathrm{TH}$ auto-correlation function (ACF) sidelobe and $C_{\max }$ denotes the maximal TH cross-correlation function (CCF) values. For aperiodic and periodic TH sequences, the theoretical bound, namely the relation between four parameters of sequences period $L$, the number of time slots $N$, TH sequences 
family size $N_{u}$ and maximal TH correlation function values $C_{\max }$ (or $S_{\max }$ ), plays an important role in construction schemes. So far, several theoretical bounds had been obtained, such as Johnson bounds and new upper bounds (Gao \& Chang, 2006; Scholtz et al, 2001).

This chapter mainly focuses on constructions and theoretical bounds of periodic TH sequences. A generalized definition of TH periodic correlation function which can be used to analytically evaluate $\mathrm{TH}$ correlation properties is presented. Based on this definition, a method to improve TH correlation properties in practical applications is proposed. By such a method, the maximum correlation function values of $\mathrm{TH}$ sequences can be reduced to a half of original values. Consequently, coincident probabilities among $\mathrm{TH}$ sequences decrease. In addition, averages of $\mathrm{TH}$ periodic correlation function values are investigated, and the relations between the averages and four TH parameters are formulated. From the results, low bounds of maximal TH correlation function values are further obtained.

In terms of the obtained low bounds, the multiple access interference (MAI) of asynchronized THSS-UWB systems is inevitable. Although orthogonal communications will be realized when accurate synchronism is held in the whole system, the accurate synchronization is difficult to be kept and catastrophic collisions will happen when synchronization in the whole system fails to be perfectly kept. In this chapter, a novel of TH sequences with zero correlation zone (ZCZ) is constructed. THSS-UWB systems employing such sequences can be without MAI when the shifts between TH sequences are in range of ZCZ. In other words, when MAI comes as small shifts of cross correlation, the MAI will be eliminated since CCF values are equal to zero in range of ZCZ. As a result, orthogonal communications can be realized while the need of accurate synchronism in whole system is reduced. The idea of approximate synchronization and ZCZ was firstly proposed for direct sequence (DS) in code division multiple access (CDMA) (Suehiro, 1994), and a lot of DSs with ZCZ had been constructed (Fan, 1999; Hayashi, 2009; Hu \& Gong, 2010). As for TH sequences with ZCZ, the corresponding studies were also presented (Guvene \& Arslan, 2004a; Guvene \& Arslan, 2004b; Zeng et al, 2011). Different from the known constructions of $\mathrm{ZCZ}$ TH sequences, the method proposed in this chapter can provide a more flexible choice of TH parameters.

Based on the analyses of TH correlation properties, multiple access performance of THSSUWB systems is presented. So far, most studies on MAI assumed that the interference was a zero-mean Gaussian random variable, namely Gaussian approximation (Canadeo et al, 2003; Durisi \& Benedetto, 2003; Hu \& Beaulieu, 2003; Mireles, 2001; Scholtz, 1993; Win \& Scholtz, 1998; Win \& Scholtz, 2000; Zhao \& Haimovich, 2002). Multiple access communication performance using UWB waveforms with TH-PPM and DS-BPSK modulations was studied (Canadeo et al, 2003), where the analyses used a fixed sequence, namely Gold codes. Based on Gaussian quadrature rules (GQR) and a characteristic function (CF) technique, two new methods to evaluate the bit error rate (BER) performance of THSS-UWB in the presence of MAI and additional white Gaussian noise (AWGN) channel were proposed (Durisi \& Benedetto, 2003; Hu \& Beaulieu, 2003), respectively. However, they still considered TH sequences as independent random variables, which will lose the practical characteristics of $\mathrm{TH}$ sequences. As a result, the effects of $\mathrm{TH}$ correlation properties on the multiple-access performance of THSS-UWB systems are ignored. In order to analyze the practical effect of $\mathrm{TH}$ sequences, this chapter derives analytical expressions of the relations between MAI 
values and $\mathrm{TH}$ correlation function values, which can be used to evaluate the BER performance in the presence of MAI.

The organization of this chapter is as follows. After the introduction, the definitions of $\mathrm{TH}$ periodic correlation function are provided in Section 2. The definitions are used to obtain theoretical bounds of TH sequences in Section 3 and improve TH correlation properties in Section 4. A novel family of TH sequences with ZCZ is obtained in Section 5. Based on TH correlation properties, the analyses of MAI are presented in Section 6. Finally, Section 7 summarizes the results.

\section{TH correlation properties}

In this section, we begin with the PPM model of THSS-UWB systems to understand how TH sequences work. We then analyze the correlation property of TH sequences in THSS-UWB systems.

\subsection{PPM model of THSS-UWB systems}

The PPM model is a kind of hopping format which is studied widely in THSS-UWB systems. In PPM model, the transmitted signal for user $i$ may be expressed as

$$
S^{(i)}(t)=\sum_{k=-\infty}^{+\infty} w\left(t-k T_{f}-c_{(k)_{L}}^{(i)} T_{c}-\delta d_{\left\lfloor k / N_{S}\right\rfloor}^{(i)}\right)
$$

where $w(\cdot)$ represents the transmitted monocycle waveform and $\left\{c_{(k)_{L}}^{(i)}\right\}$ denotes a TH sequence assigned to user $i$, where the notation $(\cdot)_{L}$ denotes a modulo $L$ operation. The TH sequence $\left\{c_{(k)_{L}}^{(i)}\right\}$ is periodic with period (or sequence length) $L$ and each sequence element is an integer in the range of $0 \leq c_{(k)_{L}}^{(i)} \leq N_{h}$. The notation $T_{f}$ denotes frame time (or pulse repetition time) and $T_{c}$ is $\mathrm{TH}$ slot time, $T_{f}=N T_{c}$, usually $N=N_{h}+1$, which represents the number of TH time slots in a frame time. The notation $\delta$ is the data shift time. The data sequence $\left\{d_{m}^{(i)}\right\}$ of user $i$ is a binary stream. One symbol may be conveyed by $N_{s}$ monocycles. The notation $\lfloor x\rfloor$ denotes the integer part of $x$.

According to the equation (1), we can see that the properties of TH sequences will play a key role in THSS-UWB systems. They ensure that UWB becomes a multiple access system and these sequences have a significant effect on synchronization and channel estimation. Fig. 1 shows how TH sequences work in THSS-UWB systems. In Fig. 1, $\left\{c_{(k)_{5}}^{(1)}\right\}=\{2,0,1,4,3\}$ and $\left\{c_{(k)_{5}}^{(2)}\right\}=\{2,4,3,0,1\}$ represent two TH sequences, respectively, where $N=L=5$. The two $\mathrm{TH}$ sequences control the position of pulse of user 1 and user 2, respectively. In addition, Fig. 1 shows that two collisions between two TH sequences emerge when some shift happens in a period. For more simplicity to be understood, time slots that happened to collision are marked with double-head arrow in Fig. 1. 


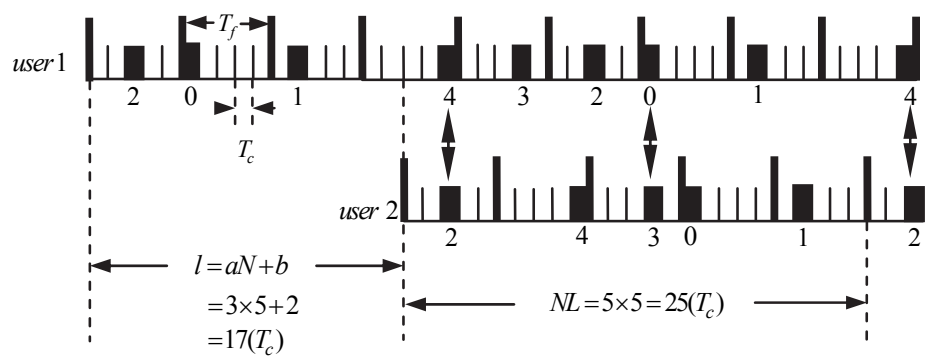

Fig. 1. The collision situation between two TH sequences (two users)

\subsection{Definitions of TH periodic correlation function}

For TH sequences, TH correlation properties are described by $\mathrm{TH}$ correlation function. In terms of chip synchronism (where shift $l \propto T_{f}$ and $0 \leq l \leq L-1$ ), TH periodic correlation function was defined as follows.

Definition 1 (Iacobucci \& Di Benedetto, 2002): Let $\left\{c_{(k)_{L}}^{(i)}\right\}$ and $\left\{c_{(k)_{L}}^{(j)}\right\}$ denote two time hopping sequences with period $L$, and then the Hamming TH periodic correlation function with shift $l$ is given by

$$
H_{i j}(l)=\sum_{k=0}^{L-1} h\left[c_{(k)_{L}}^{(i)}, c_{(k+l)_{L}}^{(j)}\right], 0 \leq l \leq L-1
$$

where

$$
h\left[c_{(k)}^{(i)},{ }^{(i)} c_{(k+l)}^{(j)}\right]=\left\{\begin{array}{ll}
1, & c_{(k)_{L}}^{(i)}=c_{(k+l)}^{(j)} \\
0, & c_{(k)_{L}}^{(i)} \neq c_{(k+l)}^{(j)} .
\end{array} .\right.
$$

According to the equation (2), we can see that Definition 1 requires $0 \leq l \leq L-1$ and $l \propto T_{f}$, that is, the frame time $T_{f}$ belonging to transmissions of different users must be synchronized. Hence, Definition 1 is the same as the definition of FH correlation function. Specially, we have $l=0$ when codeword synchronism is held in whole system.

In order to help to understand the collisions situation between the sequences, we give Fig. 2 which is array representation of sequences $\{0,3,8,10,5,1,6,9,2,4,7\}$ with $L=N=11$. In Fig. 2 , columns and rows indicate time frames and time slots, respectively. Also, each column has a unique one (black box) indicating the time slot on which we transmit according to the sequence $\left\{C_{(k)_{L}}^{(i)}\right\}$. Fig. 3 shows how Definition 1 works in the case of chip synchronism. In Fig. 3, we employ QCC sequence as an example. For QCC sequence, $c_{(k)_{L}}^{(i)}=\left(k^{2} i\right)_{p}$, where $p$ is a prime, $L=P$, the number of users $N_{u}=P-1,0 \leq k \leq L-1$ and $0 \leq i \leq P-1$. Let $p=11$, then $L=11$ and $N_{u}=10$. Fig. 3 shows the place of the maximum TH CCF value between $\left\{c_{(k)_{11}}^{(3)}\right\}$ and $\left\{c_{(k)_{11}}^{(5)}\right\}$ when $l=1$. 


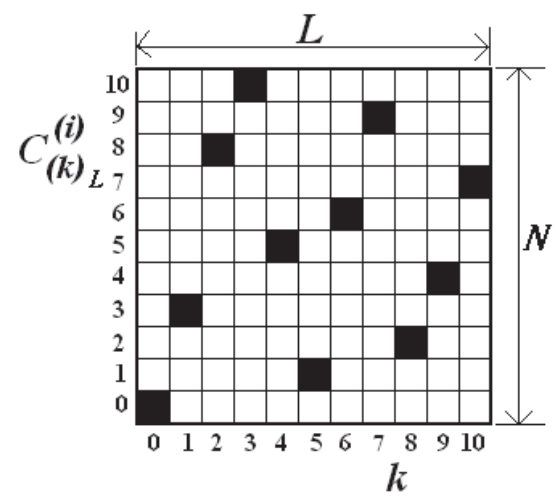

Fig. 2. Array representation of the sequence $\{0,3,8,10,5,1,6,9,2,4,7\}$, where $L=N=11$

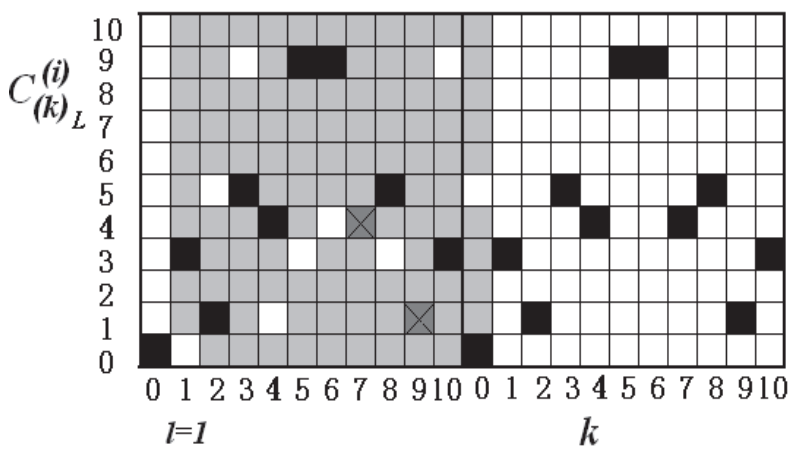

Fig. 3. The illustration of the maximum CCF value between $\left\{c_{(k)_{11}}^{(3)}\right\}$ and $\left\{c_{(k)_{11}^{(5)}}\right\}$ of QCC sequences when $l=1$, where collisions are denoted by a cross $\times$ and $C_{\max }=2$

Compared with chip synchronism and codeword synchronism which are difficulty to be kept, the asynchronism format is more interesting. Then, we consider a more general definition of TH periodic correlation function which can be used to analytically evaluate the TH correlation properties in codeword synchronism, chip synchronism and asynchronism in the whole system.

Definition 2: Let $\left\{c_{(k)_{L}}^{(i)}\right\}$ and $\left\{c_{(k)_{L}}^{(j)}\right\}$ denote two TH sequences with the period $L$, and then $\mathrm{TH}$ periodic correlation function with shift $l$ can be expressed as

$$
C_{i j}(l)=|A \cap B|,
$$

where $A=\left\{x \mid x=\left(k N+c_{(k+a)_{L}}^{(i)}\right)_{N L}\right\}, \quad B=\left\{x \mid x=\left(k N+c_{(k)_{L}}^{(j)}+b\right)_{N L}\right\}$ and $0 \leq a, b, k \leq L-1$. The notation $|x|$ represents the number of the elements in set $x$ and $A \cap B$ indicates the intersection between two sets of $A$ and $B$. The symbol $l$ denotes shift and satisfies $l=a N+b$. Then, we have $0 \leq l \leq N L-1$. 
From the equation (3), we can see that the TH periodic correlation function $C_{i j}(l)$ refers to the number of collisions between the two sequences $\left\{c_{(k)_{L}}^{(i)}\right\}$ and $\left\{c_{(k)_{L}}^{(j)}\right\}$ in a shift period of $N L$ when the shift satisfying $l=a N+b$. The smaller the value of $C_{i j}(l)$ gets, the less the number of collisions of two TH sequences gets. Then, MAI will be reduced. It should be noted that Definition 2 is different from Definition 1. The latter describes the number of collisions in terms of $l \propto T_{f}$, while the former dose in terms of $l \propto T_{c}$. In Definition 1, $0<l \leq L-1$, and in the case, the number of circular shift comparison is equal to $L$. However, in Definition 2, the number of circular shift comparison is $(N-1)$ times more than $L$, namely $N L$.

In order to demonstrate the collision situation between two TH sequences, Fig. 1 is used again. In Fig. $1, a=3$ and $b=2$, then $l=a N+b=17$. For $\left\{c_{(k)_{5}}^{(1)}\right\}$ and $\left\{c_{(k)_{5}}^{(2)}\right\}$, the set $A=\{4,8,12,15,21\}$, the set $B=\{4,11,15,17,23\}$, respectively. As a result, their TH periodic correlation function value satisfies $C_{1,2}(17)=|A \cap B|=2$.

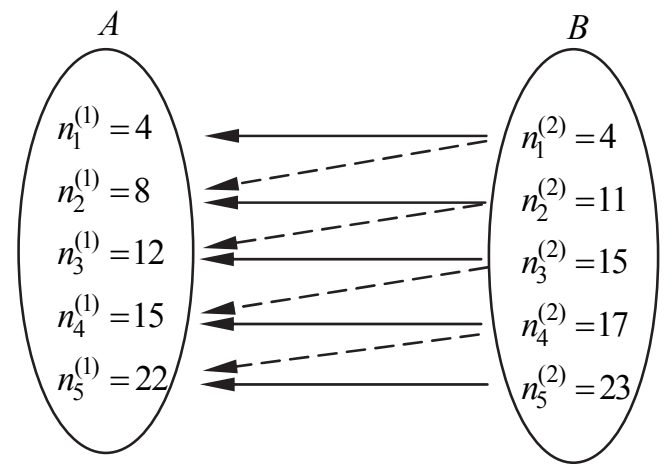

Fig. 4. The corresponding comparison between $A$ and $B$ with no shift (real line arrow) and with one frame shift (broken line arrow)

As for Definition 2, the definition of periodic correlation function of $\mathrm{TH}$ sequences is straightforward. However, it is not easy to calculate so that we need to transform the equation (3) into another equation.

We note that in the set $B, 0 \leq c_{(k)_{L}}^{(j)}+b \leq N_{h}+N-1$. When $N \leq c_{(k)_{L}}^{(j)}+b \leq N_{h}+N-1$, we have $\left(k N+c_{(k)_{L}}^{(j)}+b\right)_{N L}=\left((k+1) N+\left(c_{(k)_{L}}^{(j)}+b\right)_{N}\right)_{N L}$. Then, it is possible that the value of $\left(k N+c_{(k)_{L}}^{(j)}+b\right)_{N L}$ in the set $B$ is equal to the value of $\left((k+1) N+c_{(k+1+a)_{L}}^{(i)}\right)_{N L}$ in the set $A$. For example, when $k=2$ in Fig. 1 , we have $c_{(k)_{L}}^{(j)}+b=c_{(2)_{L}}^{(2)}+2=5$. Then, $\left(k N+c_{(k)_{L}}^{(j)}+b\right)_{N L}=\left(2 \times 5+c_{(2)_{5}}^{2}+2\right)_{5 \times 5}=15$, which is equal to the value of $\left((k+1) N+c_{(k+1+a)_{L}}^{(i)}\right)_{N L}=\left((2+1) \times 5+c_{(2+1+3)_{5}}^{(1)}\right)_{5 \times 5}=15$. Hence, there exist two cases of collision by means of corresponding comparison between $A$ and $B$ with no shift and one 
frame shift. The collisions can be depicted in Fig. 4, where the notations $\left\{n_{1}^{(1)}, \cdots, n_{5}^{(1)}\right\}$ and $\left\{n_{1}^{(2)}, \cdots, n_{5}^{(2)}\right\}$ denote time slot values of the first TH sequence and the second TH sequence, respectively.

As a result, an alternative definition of $\mathrm{TH}$ periodic correlation function can be obtained as follows.

Definition 3: Let $\left\{c_{(k)_{L}}^{(i)}\right\}$ and $\left\{c_{(k)_{L}}^{(j)}\right\}$ denote two TH sequences with period $L$, and then TH periodic correlation function with shift $l$ can be given by

$$
C_{i j}(l)=\sum_{k=0}^{L-1} h\left[\left(k N+c_{(k+a)_{L}}^{(i)}\right)_{N L},\left(k N+c_{(k)_{L}}^{(j)}+b\right)_{N L}\right]+\sum_{k=0}^{L-1} h\left[\left((k+1) N+c_{(k+1+a)_{L}}^{(i)}\right)_{N L},\left(k N+c_{(k)_{L}}^{(j)}+b\right)_{N L}\right],
$$

where the notations are the same as that in Definitions 1 and 2.

In Definition 3, it is obvious that the value of $C_{i j}(l)$ contains two parts, namely $\sum_{k=0}^{L-1} h\left[\left(k N+c_{(k+a)_{L}}^{(i)}\right)_{N L^{\prime}}\left(k N+c_{(k)_{L}}^{(j)}+b\right)_{N L}\right] \quad$ and $\quad \sum_{k=0}^{L-1} h\left[\left((k+1) N+c_{(k+1+a)_{L}}^{(i)}\right)_{N L},^{\prime}\left(k N+c_{(k)_{L}}^{(j)}+b\right)_{N L}\right]$. The two parts are suitable for $c_{(k)_{L}}^{(j)}+b<N$ and $c_{(k)_{L}}^{(j)}+b \geq N$, respectively.

In the case of $c_{(k)_{L}}^{(j)}+b<N$ and two $\mathrm{TH}$ sequences have a corresponding comparison in terms of $k$, the value of $\left((k+1) N+c_{(k+1+a)_{L}}^{(i)}\right)_{N L}$ is impossible to be equal to the value of $\left(k N+c_{(k)_{L}}^{(j)}+b\right)_{N L}$ in the second part of $C_{i j}(l)$. Similarly, in the case of $c_{(k)_{L}}^{(j)}+b \geq N$ and two TH sequences have a corresponding comparison in terms of $k$, the value of $\left(k N+c_{(k+a)_{L}}^{(i)}\right)_{N L}$ is impossible to be equal to the value of $\left(k N+c_{(k)_{L}}^{(j)}+b\right)_{N L}$ in the first part of $C_{i j}(l)$.

According to the above analyses, we can conclude that the $\mathrm{TH}$ periodic correlation function defined in Definition 3 is divided into two parts that do not include each other, and their combination is equal to the quantities of circular shift collisions in the shift period NL. Fig. 5 shows the illustration of the maximum CCF value of QCC sequences on the basis of Definition 3, where $p=11$. In comparison with Fig. 3, we can see that TH correlation properties in asynchronism format are obviously inferior to that in synchronism format.

Note that TH periodic correlation functions in previous three definitions are assumed to be discrete functions because the sequences are discrete ones. In real systems, the MAI comes as noninteger shifts of cross correlation, and then we should assume the correlation functions to be continuous.

Continuous sequences, each of which corresponds to a discrete sequence, can be defined in Definition 4. In Definition 4, each term in a discrete sequence is held within a fixed width of a continuous sequence. This allows the continuous correlation function between two continuous sequences to be easily defined, which is similar to the definition of continuous correlation function of DS (Suehiro, 1992). 


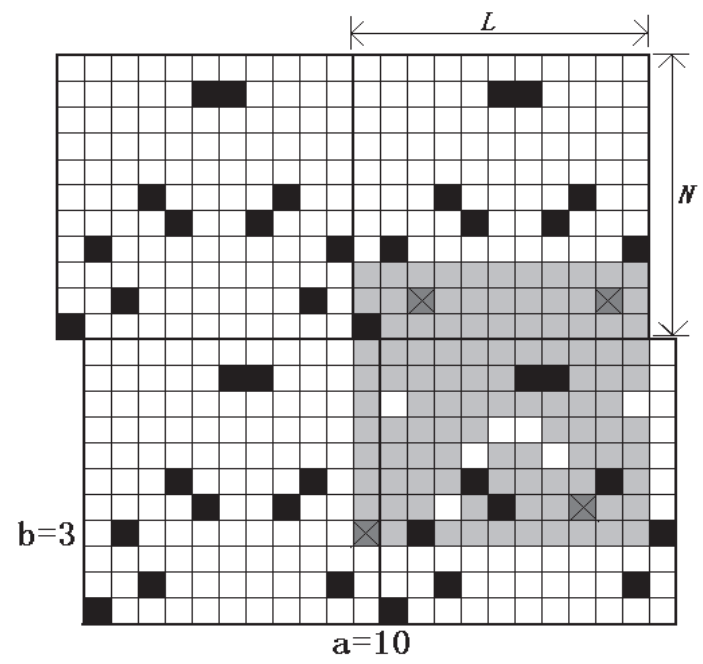

Fig. 5. The illustration of the maximum CCF value between $\left\{c_{(k)_{11}}^{(3)}\right\}$ and $\left\{c_{(k)_{11}^{(5)}}\right\}$ of QCC sequences when $N=N_{h}+1=L=11$ and $l=a N+b=113$, where collisions are denoted by a cross $\times$ and $C_{\max }=4$

Definition 4: Let $\left\{c_{(k)_{L}}^{(i)}\right\}$ and $\left\{c_{(k)_{L}}^{(j)}\right\}$ denote two TH sequences with period L. The continuous TH periodic correlation function $Y_{i j}(\tau)$ with shift term $\tau$ is defined as

$$
Y_{i j}(\tau)=(1-\alpha) \cdot C_{i j}(l)+\alpha \cdot C_{i j}(l+1),
$$

where $l$ is an integer, $\tau=l+\alpha, 0 \leq \alpha<1$, and $C_{i j}(l)$ denotes discrete TH periodic correlation function in Definition 3.

Comparing Definition 4 with Definition 3, we can see that $Y_{i j}(\tau) \leq C_{i j}(l)$. We consider the worst scenario, namely $Y_{i j}(\tau)=C_{i j}(l)$, and then we only analyze $C_{i j}(l)$ in the following sections.

\section{Theoretical bounds of TH sequences}

In this section, $\mathrm{TH}$ auto-correlation sidelobes average $\overline{C_{i i}(l)}$ and $\mathrm{TH}$ cross-correlation function average $\overline{C_{i j}(l)}$ are evaluated. Based on the obtained averages of $\overline{C_{i i}(l)}$ and $\overline{C_{i j}(l)}$, low bounds of maximal $\mathrm{TH}$ auto-correlation sidelobe $S_{\max }$ and maximal $\mathrm{TH}$ crosscorrelation function value $C_{\max }$ are obtained in the following two theorems, respectively.

Theorem 1: Let $\left\{c_{(k)_{L}}^{(i)}\right\}$ denote a TH sequence with period $L$, and then $\overline{C_{i i}(l)}$ can be expressed as

$$
\overline{C_{i i}(l)}=\frac{L^{2}-L}{N L-1}
$$


and

$$
S_{\max } \geq \frac{L^{2}-L}{N L-1}
$$

Proof: For the TH sequence $\left\{c_{(k)_{L}}^{(i)}\right\}$, the sum of TH auto-correlation function values in one shift period $N L$ can be calculated as

$$
\sum_{l=0}^{N L-1} C_{i i}(l)=L+\sum_{l=1}^{N L-1} C_{i i}(l)=L+(N L-1) \cdot \overline{C_{i i}(l)},
$$

According to Definition 3, the sum $\sum_{l=0}^{N L-1} C_{i i}(l)$ can be also expressed as

$$
\begin{aligned}
& \sum_{l=0}^{N L-1} C_{i i}(l) \\
= & \sum_{l=0}^{N L-1}\left\{\sum_{k=0}^{L-1} h\left[\left(k N+c_{(k+a)_{L}}^{(i)}\right)_{N L},\left(k N+c_{(k)_{L}}^{(i)}+b\right)_{N L}\right]+\sum_{k=0}^{L-1} h\left[\left((k+1) N+c_{(k+1+a)_{L}}^{(i)}\right)_{N L},\left(k N+c_{(k)_{L}}^{(i)}+b\right)_{N L}\right]\right\} \\
= & \sum_{l=0}^{N L-1} \sum_{k=0}^{L-1}\left\{h\left[\left(c_{(k+a)_{L}}^{(i)}\right)_{N L},\left(c_{(k)_{L}}^{(i)}+b\right)_{N L}\right]+h\left[\left(N+c_{(k+1+a)_{L}}^{(i)}\right)_{N L},\left(c_{(k)_{L}}^{(i)}+b\right)_{N L}\right]\right\} \\
= & \sum_{k=0}^{L-1} \sum_{b=0}^{N-1} \sum_{a=0}^{L-1}\left\{h\left[\left(c_{(k+a)_{L}}^{(i)}\right)_{N L},\left(c_{(k)_{L}}^{(i)}+b\right)_{N L}\right]+h\left[\left(N+c_{(k+1+a)_{L}}^{(i)}\right)_{N L}{ }^{\prime}\left(c_{(k)_{L}}^{(i)}+b\right)_{N L}\right]\right\}
\end{aligned}
$$

For the equation (9), we have $N \leq N+c_{(k)_{L}}^{(i)} \leq N+N_{h}$ and $0 \leq c_{(k)_{L}}^{(i)}+b \leq N_{h}+N-1$ since $0 \leq c_{(k)_{L}}^{(i)} \leq N_{h}$ and $0 \leq b \leq N-1$. In order to simplify the equation (9), the value of $c_{(k)_{L}}^{(i)}+b$ will be divided in two parts.

On the one hand, we have $h\left[\left(N+c_{(k+1+a)_{L}}^{(i)}\right)_{N L},\left(c_{(k)_{L}}^{(i)}+b\right)_{N L}\right]=0$ when $0 \leq c_{(k)_{L}}^{(i)}+b \leq N-1$. Then, in the equation (9),

$$
\begin{aligned}
& \sum_{a=0}^{L-1}\left\{h\left[\left(c_{(k+a)_{L}}^{(i)}\right)_{N L},\left(c_{(k)_{L}}^{(i)}+b\right)_{N L}\right]+h\left[\left(N+c_{(k+1+a)_{L}}^{(i)}\right)_{N L},\left(c_{(k)_{L}}^{(i)}+b\right)_{N L}\right]\right\} \\
& =\sum_{a=0}^{L-1} h\left[\left(c_{(k+a)_{L}}^{(i)}\right)_{N L},\left(c_{(k)_{L}}^{(i)}+b\right)_{N L}\right] \\
& =\sum_{a=0}^{L-1} h\left[c_{(k+a)_{L}}^{(i)},\left(c_{(k)_{L}}^{(i)}+b\right)_{N}\right]
\end{aligned}
$$

When $a$ is from 0 to $L-1$, the sum $\sum_{a=0}^{L-1} h\left[c_{(k+a)_{L}}^{(i)},\left(c_{(k)_{L}}^{(i)}+b\right)_{N}\right]$ represents the number of the value of $\left(c_{(k)_{L}}^{(i)}+b\right)_{N}$ appearing in one period $L$ of TH sequence $\left\{c_{(k)_{L}}^{(i)}\right\}$. 
On the other hand, when $N \leq c_{(k)_{L}}^{(i)}+b \leq N_{h}+N-1$, it is obvious that $h\left[\left(c_{(k+a)_{L}}^{(i)}\right)_{N L},\left(c_{(k)_{L}}^{(i)}+b\right)_{N L}\right]=0$. Then, in the equation (9),

$$
\begin{aligned}
& \sum_{a=0}^{L-1}\left\{h\left[\left(c_{(k+a)_{L}}^{(i)}\right)_{N L^{\prime}}\left(c_{(k)_{L}}^{(i)}+b\right)_{N L}\right]+h\left[\left(N+c_{(k+1+a)_{L}}^{(i)}\right)_{N L},\left(c_{(k)_{L}}^{(i)}+b\right)_{N L}\right]\right\} \\
& =\sum_{a=0}^{L-1} h\left[\left(N+c_{(k+1+a)_{L}}^{(i)}\right)_{N L},\left(c_{(k)_{L}}^{(i)}+b\right)_{N L}\right] \\
& =\sum_{a=0}^{L-1} h\left[N+c_{(k+1+a)_{L}}^{(i)}, N+\left(c_{(k)_{L}}^{(i)}+b\right)_{N}\right] \\
& =\sum_{a=0}^{L-1} h\left[c_{(k+1+a)_{L}}^{(i)},\left(c_{(k)_{L}}^{(i)}+b\right)_{N}\right]
\end{aligned}
$$

When $a$ is from 0 to $L-1$, the sum $\sum_{a=0}^{L-1} h\left[c_{(k+1+a)_{L}}^{(i)},\left(c_{(k)_{L}}^{(i)}+b\right)_{N}\right]$ also represents the number of the value of $\left(c_{(k)_{L}}^{(i)}+b\right)_{N}$ appearing in one period $L$ of TH sequence $\left\{c_{(k)_{L}}^{(i)}\right\}$.

According to the above analyses, we can obtain that

$$
\sum_{l=0}^{N L-1} C_{i i}(l)=\sum_{k=0}^{L-1} \sum_{b=0}^{N-1} n u m_{i}\left(\left(c_{(k)_{L}}^{(i)}+b\right)_{N}\right)
$$

where the notation $\operatorname{num}_{i}\left(\left(c_{(k)_{L}}^{(i)}+b\right)_{N}\right)$ denotes the number of the value of $\left(c_{(k)_{L}}^{(i)}+b\right)_{N}$ appearing in one period $L$ of TH sequence $\left\{c_{(k)_{L}}^{(i)}\right\}$.

For the equation (10), when $b$ is from 0 to $N-1$, the values of $\left(c_{(k)_{L}}^{(i)}+b\right)_{N}$ just constitute the set $\{0,1, \cdots, N-1\}$. Then, $\sum_{b=0}^{N-1} n u m_{i}\left(\left(c_{(k)_{L}}^{(i)}+b\right)_{N}\right)$ represents the sum of the number of every element in set $\{0,1, \cdots, N-1\}$ appearing in one period $L$ of TH sequence $\left\{c_{(k)_{L}}^{(i)}\right\}$. Hence, $\sum_{b=0}^{N-1} \operatorname{num}_{i}\left(\left(c_{(k)_{L}}^{(i)}+b\right)_{N}\right)=L$, and then $\sum_{l=0}^{N L-1} C_{i i}(l)=\sum_{k=0}^{L-1} L=L^{2}$. According to the equation (8), we have $\sum_{l=0}^{N L-1} C_{i i}(l)=L+(N L-1) \cdot \overline{C_{i i}(l)}=L^{2}$, and then $\overline{C_{i i}(l)}=\frac{L^{2}-L}{N L-1}$. As a result, we have $S_{\max } \geq \frac{L^{2}-L}{N L-1}$ since $S_{\max } \geq \overline{C_{i i}(l)}$.

\section{Q.E.D}

Similar to $\mathrm{TH}$ auto-correlation properties, low bounds of maximal $\mathrm{TH}$ cross-correlation function value $C_{\max }$ can be obtained in the following theorem.

Theorem 2: Let $\left\{c_{(k)_{L}}^{(i)}\right\}$ and $\left\{c_{(k)_{L}}^{(j)}\right\}$ denote two TH sequences with period $L$, respectively. Then $\overline{C_{i j}(l)}$ may be expressed as 


$$
\overline{C_{i j}(l)}=\frac{L}{N}
$$

and

$$
C_{\max } \geq \frac{L}{N}
$$

where $i \neq j$.

Proof: According to Definition 3, we have

$$
\begin{aligned}
& N L \cdot \overline{C_{i j}(l)} \\
& =\sum_{l=0}^{N L-1} C_{i j}(l) \\
& =\sum_{k=0}^{L-1} \sum_{b=0}^{N-1} \sum_{a=0}^{L-1}\left\{h\left[\left(c_{(k+a)_{L}}^{(i)}\right)_{N L},\left(c_{(k)_{L}}^{(j)}+b\right)_{N L}\right]+h\left[\left(N+c_{(k+1+a)_{L}}^{(i)}\right)_{N L},\left(c_{(k)_{L}}^{(j)}+b\right)_{N L}\right]\right\}
\end{aligned}
$$

The analyses of the above equation is similar to Theorem 1, and then we can obtain $N L \cdot \overline{C_{i j}(l)}=\sum_{k=0}^{L-1} \sum_{b=0}^{N-1} n u m_{i}\left(\left(c_{(k)_{L}}^{(j)}+b\right)_{N}\right)=L^{2}$ and $\overline{C_{i j}(l)}=\frac{L}{N}$. Also, it is obvious that $C_{\max } \geq \frac{L}{N}$ since $C_{\max } \geq \overline{C_{i j}(l)}$.

\section{Q.E.D}

From Theorem 1 and Theorem 2, we can see that TH correlation function averages $\overline{C_{i i}(l)}$ and $\overline{C_{i j}(l)}$ are determined by sequences period $L$ and the number of time slots $N$. When $L$ and $N$ are fixed, both $\overline{C_{i i}(l)}$ and $\overline{C_{i j}(l)}$ will be fixed for any TH sequence.

In order to explain the conclusions, we give an example. We use linear congruence codes (LCC) (Titlebaum, 1981) and QCC. For LCC sequences, $C_{(k)_{L}}^{(i)}=(k i)_{P}$, where $L=P$, $0 \leq k \leq p-1,1 \leq i \leq p-1$, and $P$ is a prime. In this example, let $P=5$ and $N=5$. Then, we have $\left\{C_{(k)_{5}}^{(1)}\right\}=\{0,1,2,3,4\}$ and $\left\{C_{(k)_{5}}^{(2)}\right\}=\{0,2,4,1,3\}$.

When $l$ is from 1 to 24 (here $N L-1=24$ ), auto-correlation sidelobes of TH sequence $\left\{C_{(k)_{5}}^{(1)}\right\}$ constitute the set $\{1,0,0,0,0,4,2,0,0,0,0,3,3,0,0,0,0,2,4,0,0,0,0,1\}$. When $l$ is from 0 to 24 , crosscorrelation values between $\left\{C_{(k)_{5}}^{(1)}\right\}$ and $\left\{C_{(k)_{5}}^{(2)}\right\}$ constitute the set $\{1,1,2,0,1,1,1,0,2,1,1,2,1,0,1,1,0,2,2,0,1,1,0,1,2\}$. Then, the averages of elements in two sets are equal to $5 / 6$ and 1 , respectively. The results correspond to $\overline{C_{1,1}(l)}=\frac{L^{2}-L}{N L-1}=\frac{5}{6}$ and $\overline{C_{1,2}(l)}=\frac{L}{N}=1$ in terms of Theorem 1 and Theorem 2.

In addition, for QCC sequences, we have $\left\{C_{(k)_{5}}^{(1)}\right\}=\{0,1,4,4,1\}$ and $\left\{C_{(k)_{5}}^{(2)}\right\}=\{0,2,3,3,2\}$. When $l$ is from 1 to 24 , auto-correlation sidelobes of $\left\{C_{(k)_{5}}^{(1)}\right\}$ constitute 
$\{0,1,0,1,1,2,1,1,0,1,1,1,1,1,1,0,1,1,2,1,1,0,1,0\}$. When $l$ is from 0 to 24 , cross-correlation values between $\left\{C_{(k)_{5}}^{(1)}\right\}$ and $\left\{C_{(k)_{5}}^{(2)}\right\}$ constitute the set $\{1,2,0,2,0,0,2,2,1,1,0,0,2,1,2,0,0,1,2,1,0,2,1,0,2\}$. Then, the averages of elements in two sets are also equal to 5/6 and 1, respectively. As a result, for any sequence, both of $\overline{C_{i i}(l)}$ and $\overline{C_{i j}(l)}$ will be fixed as long as $L$ and $N$ are fixed.

Based on Theorem 1 and Theorem 2, the further result can be also obtained. Two corollaries on TH correlation properties are expressed as follows.

Corollary 1: For a TH sequences family with period $L$, we have

$$
S_{\max }, C_{\max } \geq 1 .
$$

Corollary 2: When the period $L$ and the number of time slots $N$ are fixed, in order to obtain good TH correlation properties, correlation function values $C_{i i}(l)$ and $C_{i j}(l)$ should be close to their averages as possible.

In practice, we are also interested in maximal $\mathrm{TH}$ correlation function values $\max \left\{C_{i j}(l)\right\}$ which is the maximum of all correlation function values include cross-correlation function values and auto-correlation sidelobes. Then, the following theorem gives the low bound of $\max \left\{C_{i j}(l)\right\}$.

Theorem 3: For a TH sequences family with period $L$ and family size $N_{u}$, the average of $\mathrm{TH}$ correlation function values can be expressed as

$$
\overline{C(l)}=\frac{L^{2}\left(N_{u}+1\right)-2 L}{N L\left(N_{u}+1\right)-2}
$$

and then

$$
\max \left\{C_{i j}(l)\right\} \geq \frac{L^{2}\left(N_{u}+1\right)-2 L}{N L\left(N_{u}+1\right)-2} .
$$

Proof: For a TH sequences family with period $L$ and family size $N_{u}$, the number of autocorrelation sidelobes and the number of cross-correlation values are equal to $N_{u}(N L-1)$ and $\frac{N_{u}\left(N_{u}-1\right)}{2} N L$, respectively. Then, the number of all correlation function values without auto-correlation peak should be equal to $N_{u}(N L-1)+\frac{N_{u}\left(N_{u}-1\right)}{2} N L$.

According to the proof of Theorem 1, the sum of auto-correlation sidelobes for every TH sequence is equal to $L^{2}-L$. Then, the sum of auto-correlation sidelobes for TH sequence family is equal to $N_{u}\left(L^{2}-L\right)$.

Similarly, the sum of cross-correlation values for $\mathrm{TH}$ sequence family is equal to $\frac{N_{u}\left(N_{u}-1\right)}{2} L^{2}$. Then, the sum of all correlation function values without auto-correlation peak should be equal to $N_{u}\left(L^{2}-L\right)+\frac{N_{u}\left(N_{u}-1\right)}{2} L^{2}$.

In terms of the above analyses, we can obtain that 


$$
\overline{C(l)}=\frac{N_{u}\left(L^{2}-L\right)+\frac{N_{u}\left(N_{u}-1\right)}{2} L^{2}}{N_{u}(N L-1)+\frac{N_{u}\left(N_{u}-1\right)}{2} N L}=\frac{L^{2}\left(N_{u}+1\right)-2 L}{N L\left(N_{u}+1\right)-2} .
$$

Also, it is obvious that $\max \left\{C_{i j}(l)\right\} \geq \overline{C(l)}$ and $\max \left\{C_{i j}(l)\right\} \geq \frac{L^{2}\left(N_{u}+1\right)-2 L}{N L\left(N_{u}+1\right)-2}$.

\section{Q.E.D}

According to Theorem 3, TH correlation function average $\overline{C(l)}$ is determined by three parameters of period $L$, the number of time slots $N$ and family size $M$. When $L, N$ and $M$ are fixed, $\overline{C(l)}$ is fixed for any $\mathrm{TH}$ sequence family.

\section{Improvement of TH correlation properties}

In this section, we will provide a method that improves the correlation properties of TH sequences. Before the corresponding analyses, the maximum TH correlation function values are further analyzed according to Definition 3. We give Theorem 4 as follows.

Theorem 4: For TH sequences with period $L$, the upper bound can be given by

$$
S_{\max }, C_{\max } \leq 2 \max \left(\sum_{k=0}^{L-1} h\left[\left(c_{(k+a)_{L}}^{(i)}\right)_{N},\left(c_{(k)_{L}}^{(j)}+b\right)_{N}\right]\right) .
$$

Proof: According to the equation (4), we have

$$
C_{i j}(l)=\sum_{k=0}^{L-1} h\left[\left(c_{(k+a)_{L}}^{(i)}\right)_{N L},\left(c_{(k)_{L}}^{(j)}+b\right)_{N L}\right]+\sum_{k=0}^{L-1} h\left[\left(N+c_{(k+1+a)_{L}}^{(i)}\right)_{N L}{ }^{\prime}\left(c_{(k)_{L}}^{(j)}+b\right)_{N L}\right]
$$

We first discuss the first part of $C_{i j}(l)$, namely $\sum_{k=0}^{L-1} h\left[\left(c_{(k+a)_{L}}^{(i)}\right)_{N L},\left(c_{(k)_{L}}^{(j)}+b\right)_{N L}\right]$. Note that it operates modulo $N L$. When it operates modulo $N$, the possibility of collisions between $\left(c_{(k+a)_{L}}^{(i)}\right)_{N}$ and $\left(c_{(k)_{L}}^{(j)}+b\right)_{N}$ is larger than that of collisions between $\left(c_{(k+a)_{L}}^{(i)}\right)_{N L}$ and $\left(c_{(k)_{L}}^{(j)}+b\right)_{N L}$. Then, we have $\sum_{k=0}^{L-1} h\left[\left(c_{(k+a)_{L}}^{(i)}\right)_{N L},\left(c_{(k)_{L}}^{(j)}+b\right)_{N L}\right] \leq \sum_{k=0}^{L-1} h\left[\left(c_{(k+a)_{L}}^{(i)}\right)_{N}{ }^{\prime}\left(c_{(k)_{L}}^{(j)}+b\right)_{N}\right]$.

Similarly, the second part of $C_{i j}(l)$ satisfies

$$
\begin{gathered}
\sum_{k=0}^{L-1} h\left[\left(N+c_{(k+1+a)_{L}}^{(i)}\right)_{N L^{\prime}}\left(c_{(k)_{L}}^{(j)}+b\right)_{N L}\right] \leq \sum_{k=0}^{L-1} h\left[\left(N+c_{(k+1+a)_{L}}^{(i)}\right)_{N}{ }^{\prime}\left(c_{(k)_{L}}^{(j)}+b\right)_{N}\right] \\
=\sum_{k=0}^{L-1} h\left[\left(c_{(k+1+a)_{L}}^{(i)}\right)_{N}{ }^{\prime}\left(c_{(k)_{L}}^{(j)}+b\right)_{N}\right] .
\end{gathered}
$$

When the shift $l$ is from 0 to $N L-1$, it is obvious that

$$
\max \left(\sum_{k=0}^{L-1} h\left[\left(c_{(k+a)_{L}}^{(i)}\right)_{N},\left(c_{(k)_{L}}^{(j)}+b\right)_{N}\right]\right)=\max \left(\sum_{k=0}^{L-1} h\left[\left(N+c_{(k+1+a)_{L}}^{(i)}\right)_{N}{ }^{\prime}\left(c_{(k)_{L}}^{(j)}+b\right)_{N}\right]\right)
$$


Therefore, we have

$$
\begin{aligned}
& S_{\max }, C_{\max }=\max \left(\sum_{k=0}^{L-1} h\left[\left(c_{(k+a)_{L}}^{(i)}\right)_{N L},\left(c_{(k)_{L}}^{(j)}+b\right)_{N L}\right]+\sum_{k=0}^{L-1} h\left[\left(N+c_{(k+1+a)_{L}}^{(i)}\right)_{N L},\left(c_{(k)_{L}}^{(j)}+b\right)_{N L}\right]\right) \\
& \leq 2 \max \left(\sum_{k=0}^{L-1} h\left[\left(c_{(k+a)_{L}}^{(i)}\right)_{N},\left(c_{(k)_{L}}^{(j)}+b\right)_{N}\right]\right)
\end{aligned}
$$

\section{Q.E.D}

Based on Theorem 4, we can obtain another theorem which indicates that the correlation properties of $\mathrm{TH}$ sequences will be improved when the number of $\mathrm{TH}$ time slot satisfies $N \geq 2 N_{h}+1$.

Theorem 5: Let $\left\{c_{(k)_{L}}^{(i)}\right\}$ and $\left\{c_{(k)_{L}}^{(j)}\right\}$ denote two TH sequences with period $L$, respectively. When $N \geq 2 N_{h}+1$, we have

$$
C_{i j}(l)= \begin{cases}\sum_{k=0}^{L-1} h\left[\left(c_{(k+a)_{L}}^{(i)}\right)_{N L},\left(c_{(k)_{L}}^{(j)}+b\right)_{N L}\right], & 0 \leq b<N_{h}+1 \\ \sum_{k=0}^{L-1} h\left[\left(N+c_{(k+1+a)_{L}}^{(i)}\right)_{N L},\left(c_{(k)_{L}}^{(j)}+b\right)_{N L}\right], & N_{h}+1 \leq b<N\end{cases}
$$

and

$$
S_{\max }, C_{\max } \leq \max \left(\sum_{k=0}^{L-1} h\left[\left(c_{(k+a)_{L}}^{(i)}\right)_{N},\left(c_{(k)_{L}}^{(j)}+b\right)_{N}\right]\right) .
$$

Proof: According to the equation (4), we have

$$
C_{i j}(l)=\sum_{k=0}^{L-1} h\left[\left(c_{(k+a)_{L}}^{(i)}\right)_{N L},\left(c_{(k)_{L}}^{(j)}+b\right)_{N L}\right]+\sum_{k=0}^{L-1} h\left[\left(N+c_{(k+1+a)_{L}}^{(i)}\right)_{N L},\left(c_{(k)_{L}}^{(j)}+b\right)_{N L}\right]
$$

When $0 \leq b<N_{h}+1$, we have $0 \leq\left(c_{(k)_{L}}^{(j)}+b\right)_{N L}<2 N_{h}+1$ since $0 \leq c_{(k)_{L}}^{(j)} \leq N_{h}$. Similarly, we also have $\left(N+c_{(k+1+a)_{L}}^{(i)}\right)_{N L} \geq 2 N_{h}+1$ when $N \geq 2 N_{h}+1$. As a result, it is obvious that $\sum_{k=0}^{L-1} h\left[\left(N+c_{(k+1+a)_{L}}^{(i)}\right)_{N L},\left(c_{(k)_{L}}^{(j)}+b\right)_{N L}\right]=0 . \quad$ Then, $\quad C_{i j}(l)=\sum_{k=0}^{L-1} h\left[\left(c_{(k+a)_{L}}^{(i)}\right)_{N L},\left(c_{(k)_{L}}^{(j)}+b\right)_{N L}\right] \quad$ when $0 \leq b<N_{h}+1$.

When $N_{h}+1 \leq b<N$, we have $\left(c_{(k)_{L}}^{(j)}+b\right)_{N L} \geq N_{h}+1$ since $0 \leq c_{(k)_{L}}^{(j)} \leq N_{h}$. Combining the result with $0 \leq\left(c_{(k+a)_{L}}^{(i)}\right)_{N L} \leq N_{h}$, we can obtain $\sum_{k=0}^{L-1} h\left[\left(c_{(k+a)_{L}}^{(i)}\right)_{N L},\left(c_{(k)_{L}}^{(j)}+b\right)_{N L}\right]=0$. Hence, $C_{i j}(l)=\sum_{k=0}^{L-1} h\left[\left(N+c_{(k+1+a)_{L}}^{(i)}\right)_{N L^{\prime}}\left(c_{(k)_{L}}^{(j)}+b\right)_{N L}\right]$ when $N_{h}+1 \leq b<N$. 
According to Theorem 4, we have $S_{\max }, C_{\max } \leq \max \left(\sum_{k=0}^{L-1} h\left[\left(c_{(k+a)_{N}}^{(i)}\right)_{L},\left(c_{(k)_{L}}^{(j)}+b\right)_{N}\right]\right)$.

Q.E.D.

To show how Theorem 5 works, we give a simple example using QCC sequences, where $p=11$ and $L=11$. Fig. 6 and Fig. 7 show the distributions of correlation function values of QCC sequences when $N=11$ and $N=21$, respectively. By comparing two figures, we can see that the maximum $\mathrm{TH}$ correlation function values are deceased to a half of original values.

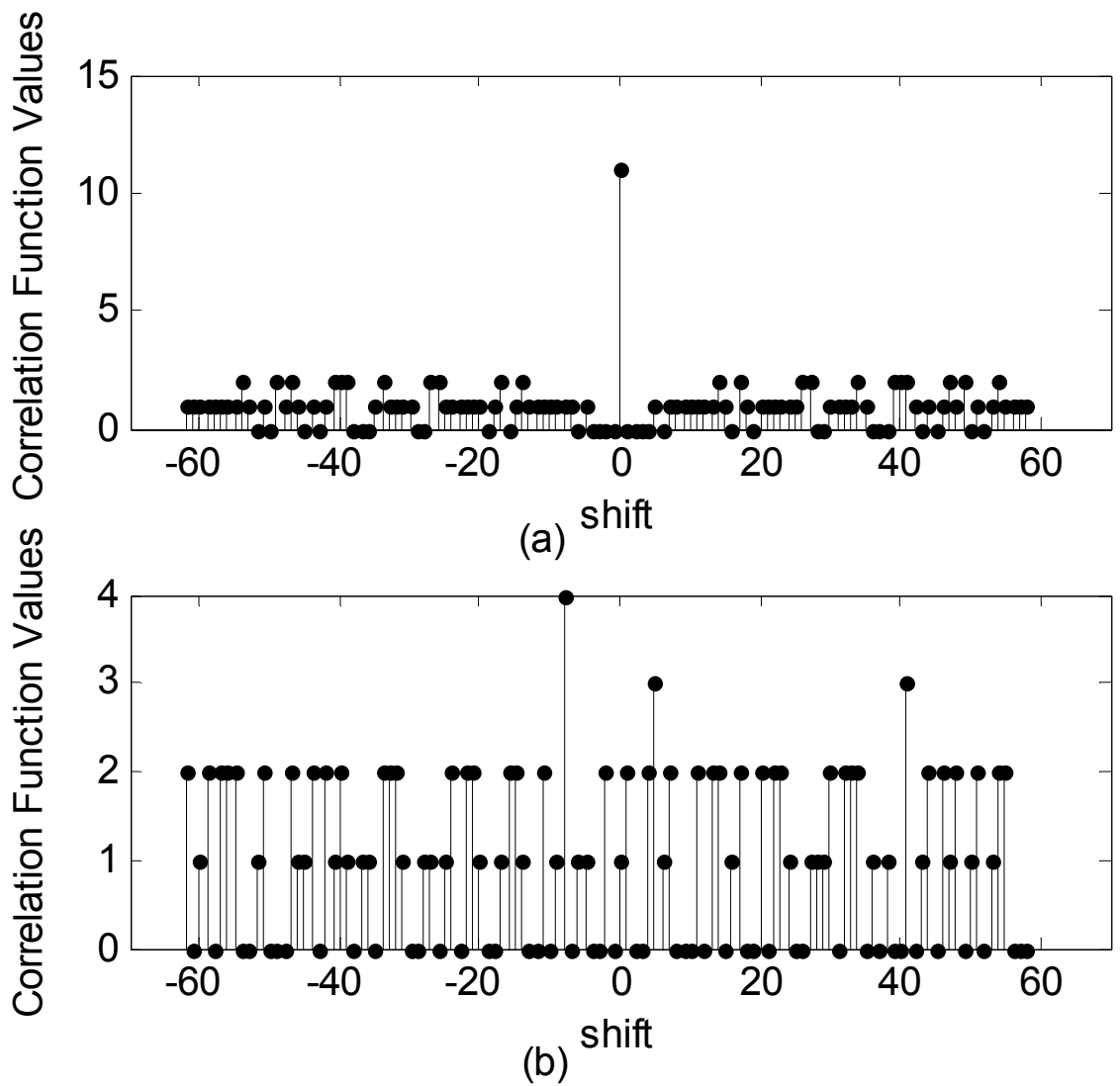

Fig. 6. The distribution of correlation function values of QCC sequences, where $N=11$. (a). ACF of $\left\{c_{(k)_{11}}^{(2)}\right\} ;(\mathrm{b})$. CCF between $\left\{c_{(k)_{11}}^{(3)}\right\}$ and $\left\{c_{(k)_{11}}^{(5)}\right\}$ 


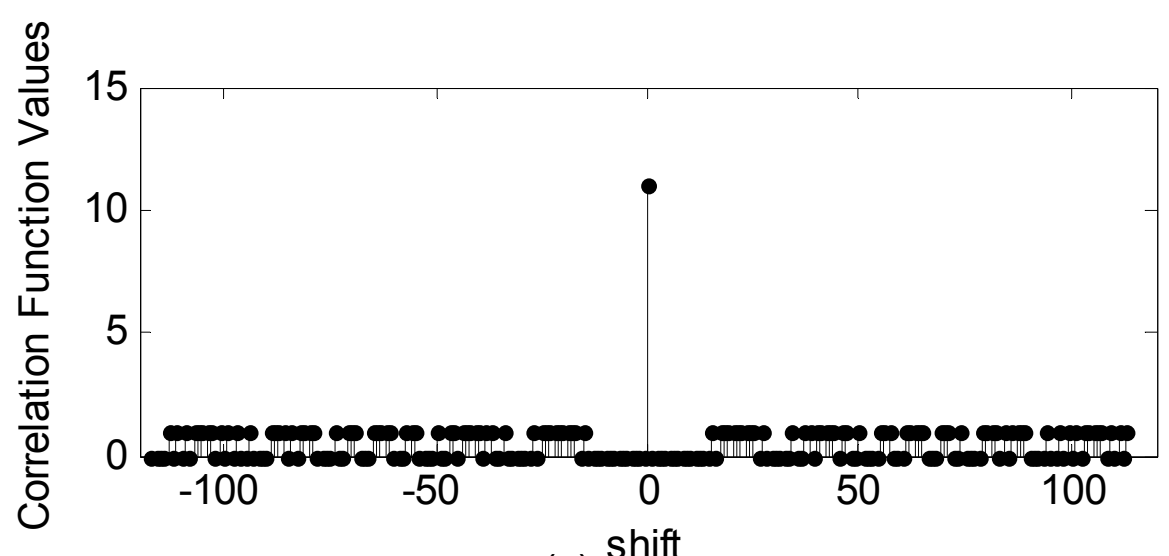

(a) shift

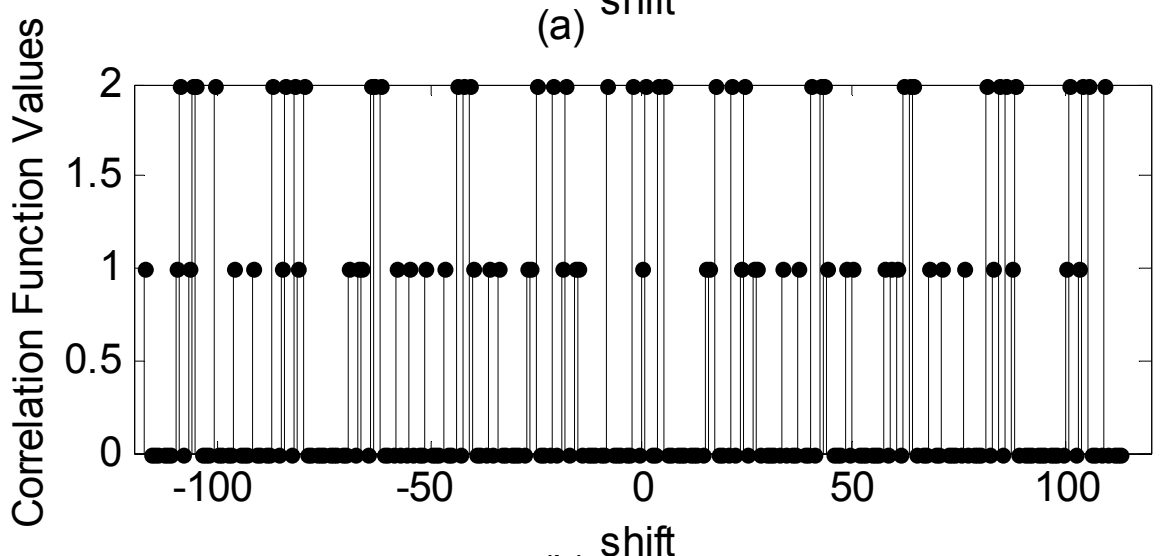

(b)

Fig. 7. The distribution of correlation function values of QCC sequences, where $N=21$. (a). ACF of $\left\{c_{(k)_{11}}^{(2)}\right\} ;(b)$. CCF between $\left\{c_{(k)_{11}}^{(3)}\right\}$ and $\left\{c_{(k)_{11}}^{(5)}\right\}$

\section{TH sequences with $\mathrm{ZCZ}$}

In this section, we begin with the definition of $\mathrm{ZCZ}$ of $\mathrm{TH}$ sequences to understand how $\mathrm{ZCZ}$ works. We then construct a class of TH sequences with $\mathrm{ZCZ}$ and prove the correlation properties of such $\mathrm{TH}$ sequences when the shifts between $\mathrm{ZCZ} \mathrm{TH}$ sequences are in the range of $Z C Z$.

\subsection{Definition of ZCZ of TH sequences}

According to Definition 3 on TH period correlation function, we can define the $\mathrm{ZCZ}$ of $\mathrm{TH}$ sequences as follows.

Definition 5: Let $C_{i j}(l)$ denotes TH periodic correlation function between two TH sequences $\left\{c_{(k)_{L}}^{(i)}\right\}$ and $\left\{c_{(k)_{L}}^{(j)}\right\}$ with period $L$, and then $\mathrm{ZCZ}$ of TH sequences can be expressed as 


$$
C_{i i}(l)= \begin{cases}L, & l=0 \\ 0, & 0<|l| \leq \frac{Z_{A C Z}}{2}\end{cases}
$$

and

$$
C_{i j}(l)=0, \quad 0 \leq|l| \leq \frac{Z_{C C Z}}{2}, \quad i \neq j,
$$

where $Z_{A C Z}$ and $Z_{C C Z}$ denote TH zero auto-correlation zone (ZACZ) width and TH zero cross-correlation zone (ZCCZ) width, respectively.

According to definition 5, both of CCF and ACF sidelobes are equal to zero when the shifts between $\mathrm{TH}$ sequences are in the range of $Z_{C Z}$, where $Z_{C Z}=\min \left\{Z_{A C Z}, Z_{C C Z}\right\}$. Then, orthogonal communications can be realized when the approximate chip synchronization is held between users in whole system.

\subsection{Construction of ZCZ TH sequences}

The principle of construction of ZCZ TH sequences can be depicted in Fig. 8, where $\left\{c_{(k)_{L}}^{(1)}\right\}=\left\{e_{(k)_{L}}^{(1)}\right\}$ and $\left\{c_{(k)_{L}}^{(2)}\right\}=\left\{N_{e h}+1+Z_{C C Z}+e_{(k)_{L}}^{(2)}\right\}$ respectively denote two TH sequences, and $\left\{e_{(k)_{L}}^{(i)}\right\}$ is any existing TH sequence satisfying $0 \leq e_{(k)_{L}}^{(i)} \leq N_{e h}$.

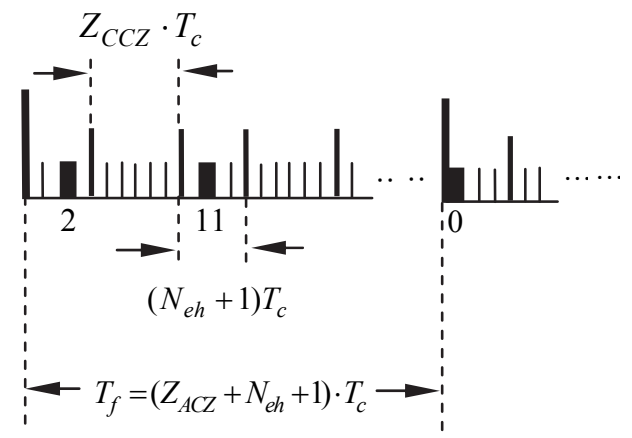

Fig. 8. The principle of construction of ZCZ TH sequences

According to Fig. 8, $c_{(0)_{L}}^{(1)}=e_{(0)_{L}}^{(1)}=2, c_{(1)_{L}}^{(1)}=e_{(1)_{L}}^{(1)}=0, e_{(0)_{L}}^{(2)}=1, N_{e h}=3$ and $Z_{C C Z}=6$. Then, we have $c_{(0)_{L}}^{(2)}=N_{e h}+1+Z_{C C Z}+e_{(0)_{L}}^{(2)}=3+1+6+1=11$. In terms of such principle, a class of $\mathrm{ZCZ} \mathrm{TH}$ sequences can be constructed as follows.

Construction of ZCZ TH Sequences: For the given $Z C Z$ width $Z_{C Z}$ which is determined by THSS-UWB systems, a novel ZCZ TH sequence $\left\{c_{(k)_{L}}^{(i)}\right\}$ can be expressed as

$$
c_{(k)_{L}}^{(i)}=(i-1)\left(N_{e h}+1+Z_{C Z}\right)+e_{(k)_{L}}^{(i)} .
$$

The widths of ZACZ and ZCCZ satisfy 


$$
Z_{A C Z}=N_{u} \cdot\left(N_{e h}+1+Z_{C Z}\right)-N_{e h}-1=N-N_{e h}-1
$$

and

$$
Z_{C C Z}=Z_{C Z}
$$

where $N=N_{u} \cdot\left(N_{e h}+1+Z_{C Z}\right)$ and $0 \leq e_{(k)_{L}}^{(i)} \leq N_{e h}$.

Based on Definition 3, correlation properties of the constructed ZCZ TH sequences can be proved as follows.

Proof: (1). We first consider the case of $i \neq j$, namely CCF.

Let the synchronization error $\lambda$ of a THSS-UWB system satisfy $|\lambda| \leq \frac{Z_{C Z}}{2} \cdot T_{c}$ when the approximate chip synchronization is held in the whole system. Correspondingly, the shift between two TH sequences is equal to $l=a N+b$, where $0 \leq a \leq L-1$ and $0 \leq b \leq Z_{C Z}$. The evaluation of $C_{i j}(l)$ will be carried out in two steps on the basis of its two components.

i. According to the equation (20), the first part of $C_{i j}(l)$ can be expressed as

$$
\sum_{k=0}^{L-1} h\left[\left(c_{(k+a)_{L}}^{(i)}\right)_{N L},\left(c_{(k)_{L}}^{(j)}+b\right)_{N L}\right]=\sum_{k=0}^{L-1} h\left[\left((i-j)\left(N_{e h}+1+Z_{C Z}\right)+\left(e_{(k+a)_{L}}^{(i)}-e_{(k)_{L}}^{(j)}\right)\right)_{N L},(b)_{N L}\right],
$$

where $-N_{e h} \leq e_{(k+a)_{L}}^{(i)}-e_{(k)_{L}}^{(j)} \leq N_{e h}$ since $0 \leq e_{(k+a)_{L}}^{(i)}, e_{(k)_{L}}^{(j)} \leq N_{e h}$. Then, it is obvious that $\left((i-j)\left(N_{e h}+1+Z_{C Z}\right)+\left(e_{(k+a)_{L}}^{(i)}-e_{(k)_{L}}^{(j)}\right)\right)_{N L} \geq 1+Z_{C Z} \quad$ when $i>j$. If $i<j$, we will have $-\left(N_{u}-1\right) \leq i-j \leq-1$. Then,

$$
\begin{aligned}
-\left(N_{u}-1\right)\left(N_{e h}+1+Z_{C Z}\right)-N_{e h} & \leq(i-j)\left(N_{e h}+1+Z_{C Z}\right)+\left(e_{(k+a)_{L}}^{(i)}-e_{(k)_{L}}^{(j)}\right) \\
& \leq-\left(N_{e h}+1+Z_{C Z}\right)+N_{e h} \\
& =-\left(1+Z_{C Z}\right) \\
& <0
\end{aligned}
$$

We can further obtain that

$$
\begin{aligned}
\left((i-j)\left(N_{e h}+1+Z_{C Z}\right)+\left(e_{(k+a)_{L}}^{(i)}-e_{(k)_{L}}^{(j)}\right)\right)_{N L} & =N L-\left|(i-j)\left(N_{e h}+1+Z_{C Z}\right)+\left(e_{(k+a)_{L}}^{(i)}-e_{(k)_{L}}^{(j)}\right)\right| \\
& \geq N L-\left(N_{u}-1\right)\left(N_{e h}+1+Z_{C Z}\right)-N_{e h} \\
& =N_{u}\left(N_{e h}+1+Z_{C Z}\right) L-\left(N_{u}-1\right)\left(N_{e h}+1+Z_{C Z}\right)-N_{e h} \\
& =N_{u}(L-1) N_{e h}+\left(N_{u} L-N_{u}+1\right)\left(1+Z_{C Z}\right) \\
& \geq 1+Z_{C Z}
\end{aligned}
$$

As a result, when $i \neq j$, we have

$$
\left((i-j)\left(N_{e h}+1+Z_{C Z}\right)+\left(e_{(k+a)_{L}}^{(i)}-e_{(k)_{L}}^{(j)}\right)\right)_{N L} \geq 1+Z_{C Z} .
$$

Due to $0 \leq b \leq Z_{C Z}$, we can obtain that 


$$
\begin{gathered}
\sum_{k=0}^{L-1} h\left[\left(c_{(k+a)_{L}}^{(i)}\right)_{N L},\left(c_{(k)_{L}}^{(j)}+b\right)_{N L}\right]=\sum_{k=0}^{L-1} h\left[\left((i-j)\left(N_{e h}+1+Z_{C Z}\right)+\left(e_{(k+a)_{L}}^{(i)}-e_{(k)_{L}}^{(j)}\right)\right)_{N L},(b)_{N L}\right] \\
=0
\end{gathered}
$$

ii. The second part of $C_{i j}(l)$ can be expressed as

$$
\begin{aligned}
& \sum_{k=0}^{L-1} h\left[\left(N+c_{(k+1+a)_{L}}^{(i)}\right)_{N L},\left(c_{(k)_{L}}^{(j)}+b\right)_{N L}\right] \\
& =\sum_{k=0}^{L-1} h\left[\left(\left(N_{u}+i-j\right)\left(N_{e h}+1+Z_{C Z}\right)+\left(e_{(k+1+a)_{L}}^{(i)}-e_{(k)_{L}}^{(j)}\right)\right)_{N L},(b)_{N L}\right]
\end{aligned}
$$

Similarly, when $i \neq j$, we obtain that $\left(\left(N_{u}+i-j\right)\left(N_{e h}+1+Z_{C Z}\right)+\left(e_{(k+1+a)_{L}}^{(i)}-e_{(k)_{L}}^{(j)}\right)\right)_{N L} \geq 1+Z_{C Z}$. Due to $0 \leq b \leq Z_{C Z}$, we can obtain that $\sum_{k=0}^{L-1} h\left[\left(N+c_{(k+1+a)_{L}}^{(i)}\right)_{N L},\left(c_{(k)_{L}}^{(j)}+b\right)_{N L}\right]=0$.

In terms of the above analyses, the CCF values of the constructed ZCZ TH sequences are equal to zero when the shifts are in range of $Z_{C C Z}$, namely $C_{i j}(l)=0$ when $0 \leq|l| \leq \frac{Z_{C C Z}}{2}$ and $i \neq j$.

(2). Secondly, we consider the case of $i=j$, namely ACF.

For an approximately synchronized THSS-UWB system, when multipath delay is in the range of $Z_{A C Z} \cdot T_{c}$, the shift of TH sequence $\left\{c_{(k)_{L}}^{(i)}\right\}$ is correspondingly equal to $l=a N+b$, where $a=0$ and $0<b \leq Z_{A C Z}$.

Similar to $C_{i j}(l)$, the evaluation of $C_{i i}(l)$ will be carried out in two steps.

i. According to equation (20), the first part of $C_{i i}(l)$ can be expressed as

$$
\sum_{k=0}^{L-1} h\left[\left((i-i)\left(N_{e h}+1+Z_{C Z}\right)+\left(e_{(k)_{L}}^{(i)}-e_{(k)_{L}}^{(i)}\right)\right)_{N L},(b)_{N L}\right]=\sum_{k=0}^{L-1} h\left[(0)_{N L},(b)_{N L}\right] .
$$

Due to $0<b \leq Z_{A C Z}$, we have $\sum_{k=0}^{L-1} h\left[\left(c_{(k+a)_{L}}^{(i)}\right)_{N L},\left(c_{(k)_{L}}^{(i)}+b\right)_{N L}\right]=\sum_{k=0}^{L-1} h\left[(0)_{N L}{ }^{\prime}(b)_{N L}\right]=0$.

ii. The second part of $C_{i i}(l)$ can be expressed as

$$
\begin{aligned}
& \sum_{k=0}^{L-1} h\left[\left(\left(N_{u}+i-i\right)\left(N_{e h}+1+Z_{C Z}\right)+\left(e_{(k+1)_{L}}^{(i)}-e_{(k)_{L}}^{(i)}\right)\right)_{N L}(b)_{N L}\right] \\
& =\sum_{k=0}^{L-1} h\left[\left(N_{u}\left(N_{e h}+1+Z_{C Z}\right)+\left(e_{(k+1)_{L}}^{(i)}-e_{(k)_{L}}^{(i)}\right)\right)_{N L},(b)_{N L}\right]
\end{aligned}
$$

Due to $-N_{e h} \leq e_{(k+1)_{L}}^{(i)}-e_{(k)_{L}}^{(i)} \leq N_{e h}$ and $N=N_{u} \cdot\left(N_{e h}+1+Z_{C Z}\right)$, we can obtain that $N-N_{e h}$ $\leq N_{u}\left(N_{e h}+1+Z_{C Z}\right)+\left(e_{(k+1)_{L}}^{(i)}-e_{(k)_{L}}^{(i)}\right) \leq N+N_{e h}$. Also, since $0<b \leq Z_{S C Z}=N-N_{e h}-1$, then, 


$$
\begin{gathered}
\sum_{k=0}^{L-1} h\left[\left(N+c_{(k+1+a)_{L}}^{(i)}\right)_{N L},\left(c_{(k)_{L}}^{(j)}+b\right)_{N L}\right]=\sum_{k=0}^{L-1} h\left[\left(N_{u}\left(N_{e h}+1+Z_{C Z}\right)+\left(e_{(k+1)_{L}}^{(i)}-e_{(k)_{L}}^{(i)}\right)\right)_{N L},(b)_{N L}\right] \\
=0
\end{gathered}
$$

According to the above analyses, the ACF sidelobes of the constructed ZCZ TH sequences are equal to zero when the shifts are in range of $Z_{A C Z}$.

Q.E.D.

\section{Effects of TH correlation properties on MAI in THSS-UWB systems}

By transforming the signal model of THSS-UWB communication systems, we obtain expressions for the relation of MAI values and $\mathrm{TH}$ correlation function values in this section,.

\subsection{Binary model of TH sequences}

According to the equation (1), we can see that only one pulse is transmitted to each user within any frame time $T_{f}$, i. e. One-Pulse-Per-Frame structure (Erseghe, 2002b; Scholtz et al, 2001). The pulse position is decided by TH sequence $\left\{c_{(k)_{L}}^{(i)}\right\}$, namely $c_{(k)_{L}}^{(i)} \cdot T_{c}$. For more easiness to understand, the structure is depicted in Fig. 9, where elements of a TH sequence are binary ones.

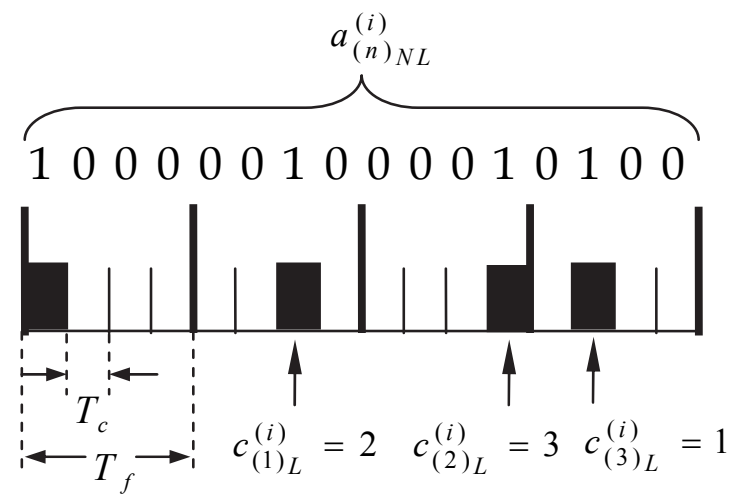

Fig. 9. The hopping format of pulses in PPM

We assume that " 1 " denotes the time slot where a pulse is modulated, and the other time slots in frame time $T_{f}$ are " 0 ". As a result, the binary TH sequence $\left\{a_{(n)_{N L}^{(i)}}\right\}$ can be obtained. The sequence $\left\{a_{(n)_{N L}}^{(i)}\right\}$ corresponds to $\left\{c_{(k)_{L}}^{(i)}\right\}$ and its period is equal to NL. According to the above analyses, the equation (1) may be transformed as

$$
S^{(i)}(t)=\sum_{n=-\infty}^{+\infty} a_{(n)_{N L}}^{(i)} \cdot w\left(t-n T_{c}-\delta d_{\left[n /\left(N \cdot N_{s}\right)\right]}^{(i)}\right),
$$


where

$$
a_{(n)_{N L}}^{(i)}=\left\{\begin{array}{ll}
1, & n=k N+c_{(k)_{L}}^{(i)} \text { for some integer } k \\
0, & \text { otherwise }
\end{array} .\right.
$$

Then, the TH periodic correlation function between $\left\{c_{(k)_{L}}^{(i)}\right\}$ and $\left\{c_{(k)_{L}}^{(j)}\right\}$ in Definition 3 can be also expressed as

$$
C_{i j}(l)=\sum_{n=0}^{L \cdot N-1} a_{(n)_{N L}^{(i)}}^{(j)} a_{(n+l)_{N L}}^{(j)}
$$

where $l$ denotes the shift between $\left\{a_{(n)_{N L}}^{(i)}\right\}$ and $\left\{a_{(n)_{N L}}^{(j)}\right\}, 0 \leq l \leq N L-1$.

Note that the equation (22) is different from the periodic correlation function of DSs. The correlation function of binary $\mathrm{TH}$ sequences describes the number of agreements to element " 1 " between sequences, called the number of collisions, where $a_{(n)_{N L}}^{(i)}$ is equal to " 0 " or " 1 " instead of " +1 " and " -1 " in ordinary correlation function, such as in DS systems. In other words, if both of $a_{(n)_{N L}}^{(i)}$ and $a_{(n)_{N L}}^{(j)}$ are " 1 " in some time slot where user $i$ and user $j$ collide, then their multiplication $a_{(n)_{N L}}^{(i)} \cdot a_{(n)_{N L}}^{(j)}$ will be also " 1 ".

As a result, the binary TH correlation function in the equation (22) refers to the number of collisions. The smaller $C_{i j}(l)$ gets, the smaller the number of collisions are, and the better TH correlation properties are.

\subsection{Multiple-access performance}

In THSS-UWB multiple-access communication systems, when $N_{u}$ links are active, the received signal $r(t)$ may be expressed as follows (Scholtz, 1993),

$$
r(t)=\sum_{i=1}^{N_{u}} A_{i} S^{(i)}\left(t-\tau_{i}\right)+n(t),
$$

where $A_{i}$ represents the attenuation of transmitter $k^{\prime}$ s signal over the propagation path to the receiver, and $\tau_{i}$ denotes time asynchronisms between the clocks of transmitter $k$ and the receiver. The notation $n(t)$ is white Gaussian receiver noise.

Without loss of generality, we assume that the receiver is interested in determining the data sent by transmitter 1 in the following analyses. We also assume that one data symbol is modulated by $L$ pulses, i. e. $N_{S}=L$, and the correlation demodulation is employed. When symbol " 0 " is sent, the shift time is zero, and the shift time is $\delta$ when symbol " 1 " is sent. Then, a template signal can be given by

$$
V(t)=\sum_{n=(k-1) \cdot N_{s} \cdot N}^{k \cdot N_{s} \cdot N-1} a_{(n)_{N L}}^{(i)} \cdot\left[w\left(t-n T_{c}\right)-w\left(t-n T_{c}-\delta\right)\right]
$$

For transmitter 1, the demodulation output of the $k^{\text {th }}$ bit is 


$$
T_{k}^{(1)}=\int_{(k-1) \cdot N_{s} \cdot N \cdot T_{c}}^{k \cdot N_{s} \cdot N \cdot T_{c}} r(t) \cdot V(t) d t
$$

Then, the received bit is decided as " 0 " when $T_{k}^{(1)} \geq 0$. Obviously, when $T_{k}^{(1)}<0$, the received bit is determined as " 1 ".

The equation (25) is also described as

$$
T_{k}^{(1)}=A_{1} N_{s}(-1)^{d_{k}^{(1)}} E_{P}+\sum_{i=2}^{N_{u}} A_{i} \cdot\left[R_{i 1}\left(\tau_{i}\right) \cdot(-1)^{d_{m}^{(i)}}+R_{i 1}^{*}\left(\tau_{i}\right) \cdot(-1)^{d_{m+1}^{(i)}}\right] \cdot E_{P}+\int_{(k-1) \cdot N_{s} \cdot N \cdot T_{c}}^{k \cdot N_{s} \cdot N \cdot T_{c}} n(t) \cdot V(t) d t
$$

where $E_{P}=\int_{-\infty}^{+\infty} w(t) \cdot[w(t)-w(t-\delta)] d t, d_{m}^{(i)}$ and $d_{m+1}^{(i)}$ represent the $m^{\text {th }}$ bit and $(m+1)^{t h}$ bit of user $i$, respectively. Transmitter 1 sends the $k^{\text {th }}$ bit. $R_{i 1}\left(\tau_{i}\right)$ and $R_{i 1}^{*}\left(\tau_{i}\right)$ denote the TH part correlation function between user $i$ and user 1 in continuous time, respectively, namely $R_{i j}(\tau)=\int_{0}^{\tau} a_{t-\tau}^{(i)} \cdot a_{t}^{(j)} d t$ and $R_{i j}^{*}(\tau)=\int_{\tau}^{L \cdot N \cdot T_{c}} a_{t-\tau}^{(i)} \cdot a_{t}^{(j)} d t$.

It is obvious that $R_{i j}(\tau)+R_{i j}^{*}(\tau)=Y_{i j}(\tau)$, where $Y_{i j}(\tau)$ is continuous TH period correlation function in Definition 4.

In the equation (26), the first part is the signal that we desire. The second part represents the MAI that the other users make to user 1, and the last part is the interference made by noise. We are interested in the second part, which will be analyzed in the following. The analysis of THSS-UWB MAI is similar to the performance evaluation for DSSS multiple-access communications (Pursley, 1977).

In order to analyze the second part of equation (26), we define now the TH aperiodic correlation function in discrete time as follows,

$$
Z_{i j}(l)= \begin{cases}\sum_{n=0}^{L \cdot N-1-l} a_{(n)_{N L}}^{(i)} \cdot a_{(n+l)_{N L}}^{(j)}, & 0 \leq l \leq L \cdot N-1 \\ \sum_{n=0}^{L \cdot N-1+l} a_{(n-l)_{N L}}^{(i)} \cdot a_{(n)_{N L}}^{(j)}, & 1-L \cdot N \leq l<0 . \\ 0, & |l| \geq L \cdot N\end{cases}
$$

According to the equation (27), in the range of $0 \leq l T_{c} \leq \tau \leq(l+1) T_{c} \leq L \cdot N \cdot T_{c}, R_{i j}(\tau)$ and $R_{i 1}^{*}\left(\tau_{i}\right)$ can be expressed as $R_{i j}(\tau)=Z_{i j}(l-L \cdot N) \cdot T_{c}+\left[Z_{i j}(l+1-L \cdot N)-Z_{i j}(l-L \cdot N)\right] \cdot\left(\tau-l T_{c}\right)$ and $R_{i j}^{*}(\tau)=Z_{i j}(l) \cdot T_{c}+\left[Z_{i j}(l+1)-Z_{i j}(l)\right] \cdot\left(\tau-l T_{c}\right)$, respectively.

Meanwhile, the TH period correlation function $C_{i j}(l)$ in the equation (22) can be expressed as $C_{i j}(l)=Z_{i j}(l)+Z_{i j}(l-L \cdot N)$, and TH period odd correlation function can be also defined as $C_{i j}^{*}(l)=Z_{i j}(l)-Z_{i j}(l-L \cdot N)$.

From the equation (26), the interference made by user $i$ with user 1 satisfies

$$
M_{i 1}\left(\tau_{i}\right)=A_{i} \cdot\left[R_{i 1}\left(\tau_{i}\right) \cdot(-1)^{d_{m}^{(i)}}+R_{i 1}^{*}\left(\tau_{i}\right) \cdot(-1)^{d_{m+1}^{(i)}}\right] \cdot E_{P} .
$$

Let $l_{i} T_{c} \leq \tau_{i} \leq\left(l_{i}+1\right) \cdot T_{c}$. When $d_{m}^{(i)}=d_{m+1}^{(i)}$, we have 


$$
\begin{aligned}
M_{i 1}\left(\tau_{i}\right) & =A_{i}\left[R_{i 1}\left(\tau_{i}\right)+R_{i 1}^{*}\left(\tau_{i}\right)\right] \cdot(-1)^{d_{m}^{(i)}} \cdot E_{P} \\
& =A_{i}\left\{\left[Z_{i 1}\left(l_{i}-L \cdot N\right)+Z_{i 1}\left(l_{i}\right)\right] \cdot T_{c}+\left[\left(Z_{i 1}\left(l_{i}+1-L \cdot N\right)+Z_{i 1}\left(l_{i}+1\right)\right)\right.\right. \\
& \left.\left.-\left(Z_{i 1}\left(l_{i}-L \cdot N\right)+Z_{i 1}\left(l_{i}\right)\right)\right] \cdot\left(\tau_{i}-l_{i} T_{c}\right)\right\} \cdot(-1)^{d_{m}^{(i)}} \cdot E_{P} \\
& =A_{i}\left\{C_{i 1}\left(l_{i}\right) \cdot T_{c}+\left[C_{i 1}\left(l_{i}+1\right)-C_{i 1}\left(l_{i}\right)\right] \cdot\left(\tau_{i}-l_{i} T_{c}\right)\right\} \cdot(-1)^{d_{m}^{(i)}} \cdot E_{P}
\end{aligned}
$$

Similarly, when $d_{m}^{(i)} \neq d_{m+1}^{(i)}$, we have

$$
\begin{aligned}
M_{i 1}\left(\tau_{i}\right) & =A_{i}\left[R_{i 1}\left(\tau_{i}\right)-R_{i 1}^{*}\left(\tau_{i}\right)\right] \cdot(-1)^{d_{m}^{(i)}} \cdot E_{P} \\
& =A_{i}\left\{C_{i 1}^{*}\left(l_{i}\right) \cdot T_{c}+\left[C_{i 1}^{*}\left(l_{i}+1\right)-C_{i 1}^{*}\left(l_{i}\right)\right] \cdot\left(\tau_{i}-l_{i} T_{c}\right)\right\} \cdot(-1)^{d_{m}^{(i)}} \cdot E_{P}
\end{aligned}
$$

The equations (28)-(29) provide the desired relation between multiple-access interference $M_{i 1}\left(\tau_{i}\right)$ and TH correlation function $C_{i j}(l)\left(\right.$ or $\left.C_{i j}^{*}(l)\right)$.

Note that $\quad\left[C_{i 1}\left(l_{i}+1\right)-C_{i 1}\left(l_{i}\right)\right]\left(\tau_{i}-l_{i} T_{c}\right)<<C_{i 1}\left(l_{i}\right) T_{c} \quad$ and $\left[C_{i 1}^{*}\left(l_{i}+1\right)-C_{i 1}^{*}\left(l_{i}\right)\right] \cdot\left(\tau_{i}-l_{i} T_{c}\right)<<C_{i 1}^{*}\left(l_{i}\right) T_{c}$. Hence, the equations (28)-(29) can be respectively expressed as $\quad M_{i 1}\left(\tau_{i}\right)=A_{i} \cdot C_{i 1}\left(l_{i}\right) \cdot T_{c} \cdot(-1)^{d_{m}^{(i)}} \cdot E_{P} \quad$ for $\quad d_{m}^{(i)}=d_{m+1}^{(i)} \quad$ and $M_{i 1}\left(\tau_{i}\right)=A_{i} C_{i 1}^{*}\left(l_{i}\right) \cdot T_{c} \cdot(-1)^{d_{m}^{(i)}} \cdot E_{P}$ for $d_{m}^{(i)} \neq d_{m+1}^{(i)}$. They describe the interference to user1 from user $i$. Furthermore, we consider a more general situation, where $N_{u}$ users are active.

Since $C_{i j}(l)=Z_{i j}(l)+Z_{i j}(l-L \cdot N)$ and $C_{i j}^{*}(l)=Z_{i j}(l)-Z_{i j}(l-L \cdot N)$, then $C_{i 1}\left(l_{i}\right) \leq C_{\max }$ and $-C_{\max } \leq C_{i 1}^{*}\left(l_{i}\right) \leq C_{\max }$. Hence, we consider the worst scenario, which happens when $C_{i 1}\left(l_{i}\right)=C_{\max }$ and $C_{i 1}^{*}\left(l_{i}\right)=C_{\max }$. Let $I_{\text {SIR }}$ denote signal-to-interference ratio (SIR) which describes the interference to user 1 from the other $N_{u}-1$ users and thermal noise, then

$$
I_{S I R}=\frac{D_{1}^{2}}{N_{1}^{2}+\sum_{i=2}^{N_{u}} M_{i 1}^{2}\left(\tau_{i}\right)}
$$

where $\quad M_{i 1}\left(\tau_{i}\right)=A_{i} \cdot C_{\max } \cdot T_{c} \cdot(-1)^{d_{m}^{(i)}} \cdot E_{P}, \quad D_{1}=A_{1} \cdot N_{s} \cdot(-1)^{d_{k}^{(1)}} \cdot E_{p}, \quad$ and $N_{1}=\int_{(k-1) \cdot N_{s} \cdot N \cdot T_{c}}^{k \cdot N_{s} \cdot N \cdot T_{c}} n(t) \cdot V(t) d t$. Then, $I_{S I R}$ can be expressed as

$$
I_{S I R}=\frac{1}{\frac{1}{I_{S N R}}+\frac{C_{\max }^{2} \cdot T_{c}^{2}}{N_{s}^{2}} \cdot \sum_{i=2}^{N_{u}}\left(\frac{A_{i}}{A_{1}}\right)^{2}},
$$

where $I_{S N R}=\frac{D_{1}^{2}}{N_{1}^{2}}$, which is a convenient parameter and equivalent to the output signal-tonoise (SNR) ratio that one might observe in single link experiments.

Then, the BER can be given by

$$
P_{e}=\frac{1}{\sqrt{2 \pi}} \int_{\sqrt{I_{S I R}}}^{\infty} \exp \left(-x^{2} / 2\right) d x .
$$


According to the equations (30)-(33), we can see that the interference and the BER are determined by $C_{\max }$ when $A_{1}, A_{i}, N_{s}, T_{c}$ and $I_{S N R}$ are specific. For the construction of TH sequences in THSS-UWB communication systems, TH correlation function values should be as small as possible so that the multiple access interference and the probability of error are small.

Similarly, for another hoping format in THSS-UWB, called PAM, the relation between multiple-access interference $M_{i 1}\left(\tau_{i}\right)$ and TH correlation function $C_{i j}(l)$ (or $C_{i j}^{*}(l)$ ) may be respectively given as $M_{i 1}\left(\tau_{i}\right)=A_{i} \cdot d_{m}^{(i)} \cdot\left\{C_{i 1}\left(l_{i}\right) \cdot T_{c}+\left[C_{i 1}\left(l_{i}+1\right)-C_{i 1}\left(l_{i}\right)\right] \cdot\left(\tau_{i}-l_{i} T_{c}\right)\right\} \cdot E_{P}$ for $d_{m}^{(i)}=d_{m+1}^{(i)} \quad$ and $\quad M_{i 1}\left(\tau_{i}\right)=A_{i} \cdot d_{m}^{(i)} \cdot\left\{C_{i 1}^{*}\left(l_{i}\right) \cdot T_{c}+\left[C_{i 1}^{*}\left(l_{i}+1\right)-C_{i 1}^{*}\left(l_{i}\right)\right] \cdot\left(\tau_{i}-l_{i} T_{c}\right)\right\} \cdot E_{P} \quad$ for $d_{m}^{(i)}=-d_{m+1}^{(i)}$, where the data $d_{m}^{(i)}$ is a binary stream (" +1 "or " -1 ") instead of " 0 " or " 1 " in PPM format.

\section{Conclusion}

The main intention of this chapter is to analyze the correlation properties of $\mathrm{TH}$ sequences for THSS-UWB systems. A more general definition of TH periodic correlation function is provided and the definition can be used to analytically evaluate the $\mathrm{TH}$ correlation properties in codeword synchronism, chip synchronism and asynchronism in the whole system. Based on the definition, theoretical bounds of $\mathrm{TH}$ sequences are presented, which relate four parameters of $L, N, N_{u}$ and $C_{\max }$ (or $S_{\max }$ ). The results can be used to evaluate the performance of $\mathrm{TH}$ sequences and provide references for the design of $\mathrm{TH}$ sequences. Also, based on the definition, a method to improve TH correlation properties in practical applications is proposed. The maximum correlation function values of $\mathrm{TH}$ sequences can be reduced to a half of original values by such a method. Specially, in terms of this method, the maximum correlation function values of QCC sequences can be reduced from $S_{\max }=2$ and $C_{\max }=4$ to $S_{\max }=1$ and $C_{\max }=2$, which achieves the best $\mathrm{TH}$ correlation properties so far in an asynchronized THSS-UWB system.

A novel TH sequence family with TH ZCZ for approximately synchronized THSS-UWB systems is constructed and its correlation properties are proved in terms of the definition of $\mathrm{TH}$ periodic correlation function presented in this chapter. When the approximate chip synchronization is held in the whole system, the MAI of THSS-UWB system employing the proposed ZCZ TH sequences is eliminated, and such THSS-UWB systems are more tolerant to the multipath problem. As a result, orthogonal communications can be realized while the need of accurate synchronism in whole system is reduced.

In addition, the multiple access performance is investigated and the relation between MAI values $I_{S I R}$ and TH correlation function values $C_{\max }$ are formulated by transforming the signal model from decimal sequence $\left\{c_{(k)_{L}}^{(i)}\right\}$ to binary sequence $\left\{a_{(n)_{N L}}^{(i)}\right\}$. Based on the obtained results, $\mathrm{TH}$ correlation function values should be as small as possible so that the multiple access interference and the probability of error are small.

\section{Acknowledgement}

We thank the National Natural Science Foundation of China (NSFC) under Grant no. 61002034 and 60872164, the Natural Science Foundation Project of CQ CSTC under Grant 
no. 2009BA2063 and 2010BB2203, Chongqing University Postgraduates' Innovative Team Building Project under Grant no. 200909B1010, and Open Research Fundation of Chongqing Key Laboratory of Signal and Information Processing (CQKLS\&IP) under Grant no. CQSIP2010-01 for supporting this work.

\section{References}

Canadeo, C.M., Temple, M.A., Boldwin, R.O. \& Raines, R.A., (2003). UWB Multiple Access Performance in Synchronous and Asynchronous Networks, Electron. Lett., Vol.39, No. 11, May 2003, pp. 880-882.

Corrada-Bravo, C., Scholtz, R. A. \& Kumar, P. V., (1999). Generating TH-SSMA Sequences with Good Correlation and Approximately Flat PSD Level, Proceedings of UWB'99, Sept. 28, 1999.

Chu, W. S.\& Colbourn, C.J., (2004). Sequence Designs for Ultra-Wideband Impulse Radio with Optimal Correlation Properties, IEEE Trans. Inf. Theory, Vol. 50, No. 10, Oct. 2004, pp. 2402-2407.

Durisi, G. \& Benedetto, S., (2003). Performance Evaluation of TH-PPM UWB Systems in the Presence of Multiuser Interference, IEEE Commun. Letters, Vol. 7, No.5, May 2003, pp. 224-226.

Erseghe, T., (2002). Ultra Wide Band Pulse Communications, Ph.D. thesis, Universita' di Padova, Italy, Feb., 2002.

Erseghe, T., (2002). Time-Hopping Patterns Derived from Permutation Sequences for Ultra Wide Band Impulse Radio Applications, Proceedings of 6th WSEAS International Conf. on Communications, July 7-14, 2002, pp.109-115.

Erseghe, T. \& Bramante, N., (2002). Pseudo-Chaotic Encoding Applied to Ultra-Wide-Band Impulse Radio, Proceedings of VTC'04F, Vol. 3, Vancouver, Canada , Dec., 2002, pp. 1711-1715.

Fan, P. Z., Suehiro, N., Kuroyanagi, N. \& Deng, X. M., (1999). Class of Binary Sequences with Zero Correlation Zone, Electron. Lett., Vol. 35, No. 10, Oct. 1999, pp. 777-779.

Gao, J. \& Chang, Y. (2006). New Upper Bounds for Impulse Radio Sequences, IEEE Trans. Inf. Theory, Vol. 52, No. 5, May 2010, pp. 2255-2260.

Guvene, I. \& Arslan, H., (2004). TH-Sequences Construction for Centralised UWB-IR Systems in Dispersive Channels, Electron. Lett., Vol. 40, No. 8, April 2004, pp. 491492.

Guvene, I. \& Arslan, H., (2004). Design and Performance Analysis of TH Sequences for UWB-IR Systems, Proceedings of WCNC'04, Atlanta, GA, USA, 2004, pp. 914-919.

Hayashi, T., (2009). A Class of Zero-Correlation Zone Sequence Set Using a Perfect Sequence, IEEE Signal Process. Lett., Vol. 16, No.4, April 2009, pp. 331-334.

Hu, B. \& Beaulieu, N. C., (2003). Exact Bit Error Rate Analysis of TH-PPM UWB Systems in the Presence of Multiple-Access Interference, IEEE Commun. Letters, Vol. 7, No. 12, Dec. 2003, pp. 572-574.

Hu, H. G. \& Gong, G., (2010). New Sets of Zero or Low Correlation Zone Sequences via Interleaving Techniques, IEEE Trans. Inf. Theory, Vol. 56, No. 4, April 2010, pp. 1702-1713.

Iacobucci, M.S. \& Di Benedetto, M.G., (2001). Time Hopping Codes in Impulse Radio Multiple Access Communication Systems, Proceedings of International Symposium on third generation Infrastructure and services, Athens, Greece, 2-3 July 2001. 
Iacobucci, M.S. \& Di Benedetto, M.G., (2002). Multiple Access Design for Impulse Radio Communication Systems, Proceedings of ICC'02, Vol. 2, Aug., 2002, pp. 817-820.

Le Martret, C. J. \& Giannakis, G. B. (2000). All-Digital PAM Impulse Radio for MultipleAccess through Frequency-Selective Multipath, Proceedings of GLOBELCOM'00, Vol. 1, San Francisco, USA, 27 Nov. - 1 Dec. 2000, pp. 77-81.

Maggio, G.M., Rulkov, N., Sushchilk, M., Tsmring, L., Volkovskii, A., Abarbanel, H., Larson, L. \& Yao, K., (1999). Chaotic Pulse Position Modulation for UltraWide-Band Communation System, Proceedings of UWB '99, Sept., 1999, pp.28-30.

Maggio, G.M., Rulkov, N. \& Reggiani, L., (2001). Pseudo-Chaotic Time Hopping for UWB Impulse Radio, IEEE Trans. on Circuits and Systems-I, Vol 48, No. 12, Dec., 2001, pp. 1424-1435.

Mireles, F. R., (2001). Performance of Ultrawideband SSMA Using Time Hopping and M-ary PPM, IEEE J. Sel. Areas Commun., Vol. 19, No. 6, June 2001, pp. 1186-1196.

Pursley, M. B., (1977). Performance Evaluation for Phase-Coded Spread-Spectrum Multiple Access Communication-Part I: System Analysis, IEEE Trans. Commun., Vol. 25, No.8, Aug. 1977, pp. 795-799.

Scholtz, R. A. (1993). Multiple Access with Time Hopping Impulse Modulation, Proceedings of MILCOM '93, Bedford, MA, USA, Oct. 11-14, 1993, pp. 447-450.

Scholtz, R. A., Kumar, P. V. \& Bravo, C. C., "Some Problems and Results in Ultra-WideBand Signal Design, Proceedings of SETA'01, May, 2001.

Suehiro, N., (1992). Elimination Filter for Co-Channel Interference in Asynchronous SSMA Systems Using Ployphase Modulatable Orthogonal Sequences, IEICE Trans. Commun., Vol. E75-B, June 1992.

Suehiro, N., (1994). A Signal Design without Co-Channel Interference for Approximately synchronized CDMA System, IEEE J. Sel. Areas Commun., SAC-12, June 1994, pp. 837-841.

Sushchick, M. Jr., Rulkov, N., Tsimring, L., Abarbanel, H., Yao, K. \& Volkovskii, A., (2000) Chaotic Pulse Position Modulation: A Robust Method of Communicating with Chaos, IEEE Commun. Letters, Vol. 4, No. 4, April, 2000, pp. 128-130.

Titlebaum. E. L., (1981). Time-Frequency Hop Signals, Part I: Coding Based upon the Theory of Linear Congruences, IEEE Trans. on Aerospace and Elect. Syst., Vol.17, No.4, July 1981, pp. 490-493.

Win, M. Z. \& Scholtz R. A. (1998). Impulse Radio: How It Works, IEEE Commun. Letters, Vol.2, No.2, Feb. 1998, pp. 36-38.

Win, M. Z. \& Scholtz R. A. (2000). Ultra-Wide Band-Width Time-Hopping Spread-Spectrum Impulse Radio for Wireless Multiple-Access Communications, IEEE Trans. on Commun., Vol. 48, No. 4, April 2000, pp. 679-690.

Zeng, Q. Peng, D. Y. \& Wang, X. N., (2011). Performance of a Novel MFSK/FHMA System Employing No-Hit Zone Sequence Set over Rayleigh Fading Channel, IEICE Trans. Fundamentals, Vol. E94-A, No. 2, Feb. 2011, pp. 526-532.

Zhao, L. \& Haimovich, A. M., (2002). The Capacity of an UWB Multiple-Access Communications System, Proceedings of ICC'02, Vol.3, 2002, pp.1964-1968. 


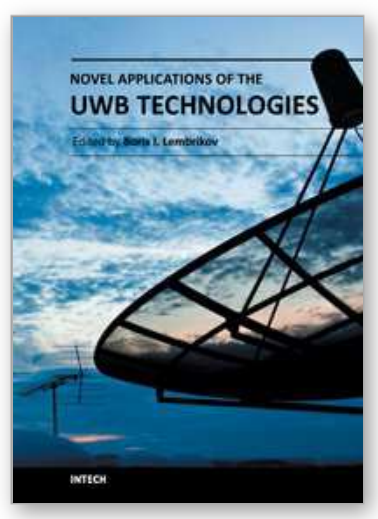

\author{
Novel Applications of the UWB Technologies \\ Edited by Dr. Boris Lembrikov
}

ISBN 978-953-307-324-8

Hard cover, 440 pages

Publisher InTech

Published online 01, August, 2011

Published in print edition August, 2011

Ultra wideband (UWB) communication systems are characterized by high data rates, low cost, multipath immunity, and low power transmission. In 2002, the Federal Communication Commission (FCC) legalized low power UWB emission between $3.1 \mathrm{GHz}$ and $10.6 \mathrm{GHz}$ for indoor communication devices stimulating rapid development of UWB technologies and applications. The proposed book Novel Applications of the UWB Technologies consists of 5 parts and 20 chapters concerning the general problems of UWB communication systems, and novel UWB applications in personal area networks (PANs), medicine, radars and localization systems. The book will be interesting for engineers and researchers occupied in the field of UWB technology.

\title{
How to reference
}

In order to correctly reference this scholarly work, feel free to copy and paste the following:

Zhenyu Zhang, Fanxin Zeng, Lijia Ge and Guixin Xuan (2011). Time-Hopping Correlation Property and Its Effects on THSS-UWB System, Novel Applications of the UWB Technologies, Dr. Boris Lembrikov (Ed.), ISBN: 978-953-307-324-8, InTech, Available from: http://www.intechopen.com/books/novel-applications-of-the-uwbtechnologies/time-hopping-correlation-property-and-its-effects-on-thss-uwb-system

\section{INTECH}

open science | open minds

\section{InTech Europe}

University Campus STeP Ri Slavka Krautzeka 83/A 51000 Rijeka, Croatia Phone: +385 (51) 770447 Fax: +385 (51) 686166 www.intechopen.com

\section{InTech China}

Unit 405, Office Block, Hotel Equatorial Shanghai No.65, Yan An Road (West), Shanghai, 200040, China 中国上海市延安西路65号上海国际贵都大饭店办公楼405单元 Phone: +86-21-62489820

Fax: +86-21-62489821 
(C) 2011 The Author(s). Licensee IntechOpen. This chapter is distributed under the terms of the Creative Commons Attribution-NonCommercialShareAlike-3.0 License, which permits use, distribution and reproduction for non-commercial purposes, provided the original is properly cited and derivative works building on this content are distributed under the same license. 